\title{
Line $\times$ Tester Analysis for Yield and 100-grain Weight Under Normal and Water Stress Conditions in Yellow Maize (Zea mays L.)
}

\author{
Sayed, K.A.K. ${ }^{1}$; ${ }^{*}$ M.B. Ali ${ }^{1}$; Kh. A.M. Ibrahim ${ }^{2}$; K.A. Kheiralla ${ }^{1}$
} and M.Z. EL-Hifny ${ }^{1}$

\author{
${ }^{1}$ Agronomy Department, Faculty of Agricultural, Assiut University \\ ${ }^{2}$ Agronomy Department, Faculty of Agricultural, New Valley University \\ *Corresponding author email: mali@aun.edu.eg
}

Received on: $18 / 2 / 2020$

Accepted for publication on: 26/2/2020

\begin{abstract}
Maize (Zea mays L.) is the third most important cereal grain in Egypt (after wheat and rice) but it is vulnerable to water stress which causes lead losses in both yield and quality. In the current study we evaluated $100 \mathrm{~S} 1$-lines along with their top-crosses using two testers under normal and water stress conditions. We used line $\times$ tester to assess general (GCA) and specific (SCA) combining ability effects for 100-grain weight and grain yield per plot; In addition, we estimated heritability for both traits. Result of line $\times$ tester analysis showed highly significant differences among parents, crosses vs parents., In addition, between testers and lines $\times$ testers for both traits under normal and water stress condition. The results, lines showed non-significant for 100-grain weight under normal and water stress condition. Grain yield per plot showed non-significant differences under normal condition while it showed significant under water stress condition. Under normal condition, S1-lines 56 and 88 possessed the highest 100-grain weight while, the highest values of 100-grain weight were found in cross combinations including S1-line $29 \times \mathrm{SC} 162$ and S1-line 61× TWC352. On the other hand, S1-lines 65 and 68 possessed the highest grain yield per plot while, the highest values of grain yield per plot were found in cross combinations including S1-line $86 \times$ SC162 and S1-line 37× TWC352. Under normal condition, S1-lines 10 and 99 displayed positive and significant GCA effects for 100-grain weight while, S1-line 86 and 55 exhibited the maximum GCA effect for grain yield per plot. Top-crosses including S1-line $29 \times$ SC162 and S1-line $75 \times \mathrm{TWC} 352$ were good specific combiners for 100-grain weight while, the top-crosses including S1-line $29 \times$ SC162 and S1-line $78 \times$ TWC352 were good specific combiners for grain yield per plot. Heritability in broad sense showed moderate highly estimates for both traits. Our results indicated the preponderance of dominance gene action in controlling both aforementioned traits. In conclusion, these S1-lines are promising to produce drought tolerant inbred line in the future, which may lead to produce drought tolerant hybrid.
\end{abstract}

Keywords: General and specific combining ability, heritability, line $\times$ tester, maize, water stress

\section{Introduction}

Zea mays L. is one of the most important cereal crop in the world after wheat and rice Reddy et al.(2012). It is used as food for human and feed for livestock. In 2017 season, the harvested area in the world was 239,614,584 Ha producing 1,393,981,150 MT. The harvested area in Egypt was 920,601 ha produc- 
ing 7,100,000 MT (FAO, 2017). To meet the ever-increasing demand, maize production can be increased by application of improved agronomic techniques to get varieties with higher qualitative and quantitative traits and resilience to abiotic stresses (Ali et al. 2014). Drought is a serious problem, which dramatically decreases crop yield and quality. Drought injury may be alleviated by developing droughttolerant hybrids adapted to dry environments such as found in the new reclaimed lands. Khalili et al. 2013 emphasized that drought stress is one of the most important abiotic stresses, which reduces growth, development and production of plants. Yue et al. (2018) found that drought had substantial effects on maize yield as well as other agronomic traits. Briefly, they stated that under water stress, number of rows per ear and 1000grain weight are efficient traits for screening for drought-tolerant genotypes in maize. Moreover, Khan et al. (2001) found reduction in yield and its components including 1000-grain weight under water stress. Furthermore, Zamaninejad et al. (2013) reported that drought stress caused significant reduction in grain yield, number of rows per ear, number of grains per row, ear diameter and ear length. The deleterious effects of drought stress on yield attributes, e.g. number of rows per ear, number of grains per row and reduced grain size, cause reduction in yield. Combining ability is a powerful tool that has been extensively used to detect the best combiner parents in a series of its crosses and provides information on the nature and magnitude of gene actions (Uddin et al. 2008). There are two constituents of combining ability including general combining ability (GCA) and specific combining ability (SCA). Firstly, GCA is the average performance of parents in a series of crosses, while SCA describes those cases in which certain combinations perform relatively better or worse than would be expected on the basis of average performance of parents (Hundera 2017). Therefore, both constituents of combining ability are informative estimates for breeders in any breeding programs, which aids in identifying the best combinations of parents. Sadalla et al. (2017) Found that GCA and SCA mean squares were highly significant for some traits, but the mean squares for reciprocal combining ability were highly significant for 100-grain weight. In addition, Rahman et al. (2018) stated that crosses displayed positive SCA effects for 100-grains weight and grain yield could possibly be utilized in maize breeding programs for developing high yielding maize hybrids accompanied with other desirable attributes. Khattab et el. (2011) found that general combing ability (GCA) effect indicated that some lines under normal and drought stress condition seem to be good general combiners for increasing yield and yield components of hybrids. The work of Ojo et al. (2007) empahsized that GCA mean squares were highly significant for grain yield. Emyhum (2013) stated that Specific combining ability variance was important than general combining ability variance for all traits indicating preponderance of dominance variance in normalling these characters. Bekele et al. (2014) found high heritability estimates for 
both 100-grain weight and grain yield per plot. Furthermore, they detected high genetic advance juxtaposed with high heritability for grain yield per plot. Rafiq et al. (2010) reported that most of the traits had high heritability estimates indicating the preponderance of additive gene action; in addition, grain yield per plant and number of grains per row represents additive gene effects. Line $\times$ tester mating approach is an effective tool; which permits the inclusion of a set of entries to estimate combining ability, gene action, male and female, and aid to identify desirable parents and crosses (Hundera 2017). Amin et al. (2014) reported that Line $\times$ tester analysis is an important method randomly used to evaluate the inbred lines. Through line $\times$ tester analysis about $50 \%$ of the inbred lines can be eliminated (Singh and Chaudhary 1985). Genetic information was obtained by different quantitative genetic methods line $\mathrm{x}$ tester analysis is a suitable and efficient method with eligible speed (Singh and Chaudhary, 1985). The line $\mathrm{x}$ tester analysis method has been widely used by plant breeders (Aly, 2013). The objectives of the current study were to: 1) elucidate the effect of water stress on yield and 100-grain weight in 100 $\mathrm{S} 1$ selected lines, 2) estimate genetic variances and heritability and 3 ) conduct line $\times$ tester analysis and estimate general and specific combining abilities for these lines using two testers, namely, SC162 and TWC352.

\section{Materials and Methods Growing condition}

This study was carried out during the period from 2018 to 2019 at Assiut University Farm using one maize population (IY376, imported from India 1969) to study the effect of water stress on agronomic traits, line $\times$ tester interaction and combining ability in maize using $100 \mathrm{~S}_{1}$ lines.

In March 2018, the population was grown, 300 vigorous and disease free plants were selected before silking, and self-pollinated. After harvest, 100 selfed ears (S1's) which had sufficient grains were chosen. Selected S1ears were individually shelled and every S1-line was divided into four equal parts. In August 2018, topcrosses were formed for S1-lines using two testers, single cross 162(SC162) and three way cross 352 (TWC352). In 2019 season, 100 S1lines and their top-crosses with SC162 and TWC 352 testers (302 genotype entry) were evaluated at Assiut University Agricultural Research Station under normal condition (every 10 days) and water stress condition (irrigated every 20 days after $2^{\text {nd }}$ irrig.) using simple lattice design $(10 \times 10)$ with three replications. The experimental plot was one row; 3 meters long with $70 \mathrm{~cm}$ between rows, planting was in hills spaced 30 $\mathrm{cm}$ apart. Seedlings were thinned at one plant/hill before the first condition. Fertilizers were applied at the rate of $120 \mathrm{~kg}$ nitrogen/fed., before the first and second conditions. Other cultural practices were carried out as usual during the season.

\section{Traits Studied}

Grain yield /plot in grams (gms): weight of grain yield of each plot (10 plants) adjusted to $15.5 \%$ moisture content. The 100-grain weight in grams (gms): weight of 100-grains weight were randomly 
sampled from the aforementioned trait.

\section{Statistical analysis}

Statistical analyses were conducted using $\mathrm{R}$ software (version 3.6.1) utilizing package AGRICOLAE (De Mendiburu 2014).

Line $(\mathrm{L}) \times$ tester $(\mathrm{T})$ analysis were performed using the method described by Kempulorne (1957). General (GCA) and specific (SCA) combining ability effects were calculated for grain yield and 100-grain weight. The statistical model used to obtain the different effects was as follows:

$$
Y_{i j k}=\mu+\rho_{k}+l_{i}+t_{j}+(l \times t)_{i j}+\varepsilon_{i j k}
$$

Where: $Y_{i j k}=$ the value of a character measured on $i^{\text {th }} \times j^{\text {th }}$ progeny in $\mathrm{k}^{\text {th }}$ replication, $\mu$ is the general mean, $\rho_{k}$ effect of $\mathrm{k}^{\text {th }}$ replication, $l_{i}$ is the effect of the $\mathrm{i}^{\text {th }}$ line, $t_{j}$ is the effect of the $\mathrm{j}^{\text {th }}$ tester, $(l \times t)_{i j}$ is the interaction effect of the cross between $i^{\text {th }}$ line and $\mathrm{j}^{\text {th }}$ tester and $\varepsilon_{i j k}$ is the error term associated with each observation. Combined analysis of both normal and water stress conditions was not performed due lack of homogeneity of variances according to Bartlett $\mathrm{s}$ test (Bartlett 1937).

The combing ability ratio (CAR) was utilized, in the current study, to anticipate the gene action as per Baker (1978) using the following equation:

$$
C A R=2 \sigma_{G C A}^{2} /\left(2 \sigma_{G C A}^{2}+\sigma_{S C A}^{2}\right),
$$

where $\sigma_{G C A}^{2}$ and $\sigma_{S C A}^{2}$ are the GCA and SCA variances, respectively.

Heritability in broad sense $\left(\mathrm{h}_{\mathrm{b}}\right)$ for both traits was estimated according to Singh and Chaudhary (1985) using the following formula: $\mathrm{h}_{\mathrm{b}}=\frac{\sigma^{2} g}{\sigma^{2} p}$

Where:

$\sigma^{2} g=$ genetic variance, and $\sigma^{2} p=$ phenotypic variance.

\section{Results and Discussion}

Line $\times$ Tester analysis

One hundred S1-lines were crossed with two testers (SC162 and TWC 352) resulted in 200 top-crosses. The lines, testers and top-crosses were evaluated under normal and water stress condition to identify the best drought-tolerant crosses. Analysis of variance for the studied traits under normal and water stress condition are presented in Table (1). Under normal condition, the results showed significant differences for 100-grain weight and grain yield per plot for parents and parents vs crosses. In addition, the crosses showed significant differences for both traits. For lines, both traits revealed non-significant differences. For testers, significant differences were found in both traits. Finally, line $\times$ tester of both traits showed significant differences. The greater contributions of lines $\times$ tester interaction than testers for both studied traits showed higher estimates of variance due to specific combining ability. In this context, our results were consistent with those found by Akula et al. (2018)

Results exhibited significant differences under water stress condition for 100-grain weight and grain yield per plot for parents and parents vs crosses; in addition, the crosses showed significant differences for both studied traits. For lines, 100grain weight showed non-significant differences; whereas, grain yield per 
plot exhibited significant differences.

For testers, significant differences were found in both studied traits. Finally, for line $\times$ tester, all traits showed significant differences. The greater contributions of lines $\times$ tester interaction than testers for both studied traits showed higher estimates of variance due to specific combining ability, in this regard, Akula et al. (2018) reported similar results.

Table 1. Mean squares of line $\times$ tester analysis for 100 -grain weight and grain yield per plot under normal and water stress conditions.

\begin{tabular}{|l|c|c|c|c|c|}
\hline \multirow{2}{*}{ Source } & \multirow{2}{*}{ DF } & \multicolumn{4}{|c|}{ MS } \\
\cline { 3 - 6 } & & \multicolumn{2}{|c|}{ Normal condition } & \multicolumn{2}{c|}{ Water stress condition } \\
\cline { 3 - 6 } & & $\mathbf{1 0 0 - G W}$ & $\mathbf{G Y}$ & $\mathbf{1 0 0 - G W}$ & GY \\
\hline Replications & 2 & $1.02^{\mathrm{NS}}$ & $83579.26^{* *}$ & $3.83^{\mathrm{NS}}$ & $51532.76^{* *}$ \\
\hline Genotypes & 301 & $19.41^{* *}$ & $200654.15^{* *}$ & $24.41^{* *}$ & $172844.67^{* *}$ \\
\hline Parents & 101 & $21.07^{* *}$ & $184517.47^{* *}$ & $21.50^{* *}$ & $85407.29^{* *}$ \\
\hline Parents vs. Crosses & 1 & $1474.27^{* *}$ & $29304956.27^{* *}$ & $2976.76^{* *}$ & $33000040.26^{* *}$ \\
\hline Crosses & 199 & $11.26^{* *}$ & $62591.36^{* *}$ & $11.05^{* *}$ & $52261.65^{* *}$ \\
\hline Lines & 99 & $10.73^{\text {NS }}$ & $59846.23^{\text {NS }}$ & $10.23^{\text {NS }}$ & $55517.75^{*}$ \\
\hline Testers & 1 & $196.69^{* *}$ & $996795.66^{* *}$ & $345.54^{* *}$ & $1073076.94^{* *}$ \\
\hline Lines $\times$ Testers & 99 & $9.92^{* *}$ & $55900.08^{* *}$ & $8.48^{* *}$ & $38694.29^{* *}$ \\
\hline Error & 602 & 2.19 & 8567.16 & 2.11 & 9294.06 \\
\hline contribution of lines & & 47.39 & 47.57 & 46.07 & 52.85 \\
\hline contribution of tester & & 8.77 & 8.00 & 15.72 & 10.32 \\
\hline contribution of line $\times$ tester & & 43.83 & 44.43 & 38.31 & 36.83 \\
\hline$* * *$
\end{tabular}

, ${ }^{* *}$ significant at 0.05 and 0.01 levels of probability, respectively.

$100-\mathrm{GW}=100$-grain weight $(\mathrm{g}), \mathrm{GY}=$ grain yield per plot $(\mathrm{g})$.

\section{Mean performance}

Mean performance of $100 \mathrm{~S} 1$ lines for 100-grain weight evaluated under normal condition are presented in Table (2). Data exhibited that S1lines 56 and 88 possessed the highest 100-grain weight while S1-lines 17 and 52 had the lowest 100-grain weight. For the tester, SC162 it had higher 100-grain weight than TWC352. Mean performances for 100-grain weight of 200 top-crosses under normal condition are presented in Table (3). Results showed that the top-crosses involving TWC352 had higher 100-grain weight than those involving SC162. The highest values of 100-grain weight were found in cross combinations including S1-line $29 \times$ SC162 and S1-line $99 \times$ SC162; in addition to S1-line $31 \times$ TWC352 and S1-line $61 \times$ TWC352. Whereas, the lowest 100-grain weight values were observed in cross combinations including S1-line $55 \times \mathrm{SC} 162$ and S1-line $68 \times \mathrm{SC162}$; in addition to S1-line $17 \times$ TWC352 and S1-line 29× TWC352. Under water stress condition mean performance of 100 S1-lines for 100-grain weight are shown in Table (4). Data displayed that the highest values of 100-grain weight were observed in S1-lines 34 and 86 unlike S1-lines 55 and 93. For the tester, TWC352 have lower 100grain weight than SC162. Under water stress condition the mean performance of 200 top-crosses for 100grain weight are presented in Table (5). Results showed that the topcrosses including TWC352 had higher 100- grain weight than those 
involving SC162. The highest values of 100-grain weight were observed in cross combinations including S1-line $10 \times \mathrm{SC} 162$ and S1-line $34 \times \mathrm{SC} 162$; in addition to S1-line $84 \times$ TWC 352 and S1-line $98 \times$ TWC 352 . Whereas, the lowest values of 100- grain weight were found in cross combinations including S1-line $52 \times \mathrm{SC} 162$ and S1line $75 \times \mathrm{SC} 162$; in addition to S1-line 29x TWC352 and S1-line $95 \times$ TWC352.

Mean performance of 100 S1lines for grain yield per plot evaluated under normal condition are presented in Table (6). Data exhibited that S1-lines 65 and 68 possessed the highest grain yield per plot while: S1lines 63 and 77 had the lowest grain yield per plot. For the tester, SC162 had higher grain yield per plot than TWC352. Mean performances for grain yield per plot of 200 top-crosses under normal condition are presented in Table (7). Results showed that the top-crosses involving TWC352 had higher grain yield per plot than those involving SC162.The highest values of grain yield per plot found in cross combinations including S1 line $2 \times$ SC162 and S1 line $86 \times \mathrm{SC} 162$; in addition to S1-line $37 \times$ TWC352 and S1-line $99 \times$ TWC352. However, the lowest grain yield per plot values were observed in cross combinations including S1-line $73 \times \mathrm{SC} 162$ and S1-line $85 \times \mathrm{SC} 162$; in addition to S1-line 9xTWC352 and S1-line 27× TWC352.

Under water stress condition the mean performance of 100 S1-lines for grain yield per plot are shown in Table (8). Data displayed that the highest values of grain yield per plot were observed in S1-lines 76 and 88 unlike, S1-line 3 and 89. For the tester, TWC352 have lower grain yield per plot than SC162.Under water stress condition mean performance of 200 top-crosses for grain yield per plot are presented in Table (9). Results showed that the topcrosses including TWC352 had higher grain yield per plot than those involving SC162.The highest values of grain yield per plot found in cross combinations including S1-line $34 \times \mathrm{SC} 162$ and S1-line $98 \times \mathrm{SC} 162$; S1-line 70×TWC352 and S1-line 98× TWC352. Whereas, the lowest values of grain yield per plot were found in the cross combinations including S1line $50 \times \mathrm{SC} 162$ and S1-line $87 \times$ SC162; in addition to S1-line $4 \times$ TWC352 and S1-line 53×TWC352. 
Website: www.aun.edu.eg/faculty_agriculture/journals_issues_form.php E-mail: ajas@aun.edu.eg

Table 2. Means (M) and their standard error (SE) of 100-grain weight (g) for parents (S1-lines and two testers) under normal condition.

\begin{tabular}{|c|c|c|c|c|c|}
\hline S1-line & $\mathbf{M} \pm \mathbf{S E}$ & S1-line & $\mathbf{M} \pm \mathbf{S E}$ & S1-line & $\mathbf{M} \pm \mathbf{S E}$ \\
\hline 1 & $20.41 \pm 0.88$ & 35 & $19.98 \pm 1.07$ & 69 & $17.22 \pm 1.35$ \\
\hline 2 & $26.10 \pm 1.18$ & 36 & $19.00 \pm 0.77$ & 70 & $17.09 \pm 0.12$ \\
\hline 3 & $20.23 \pm 1.02$ & 37 & $20.88 \pm 0.18$ & 71 & $19.04 \pm 1.12$ \\
\hline 4 & $21.89 \pm 0.63$ & 38 & $20.86 \pm 1.37$ & 72 & $22.44 \pm 1.08$ \\
\hline 5 & $19.94 \pm 0.31$ & 39 & $22.59 \pm 0.46$ & 73 & $20.50 \pm 1.87$ \\
\hline 6 & $16.77 \pm 0.89$ & 40 & $24.59 \pm 0.30$ & 74 & $23.33 \pm 1.45$ \\
\hline 7 & $21.18 \pm 2.06$ & 41 & $19.54 \pm 0.74$ & 75 & $19.35 \pm 0.88$ \\
\hline 8 & $18.32 \pm 0.44$ & 42 & $20.68 \pm 0.43$ & 76 & $20.07 \pm 0.66$ \\
\hline 9 & $20.43 \pm 1.30$ & 43 & $19.95 \pm 0.55$ & 77 & $21.26 \pm 1.01$ \\
\hline 10 & $24.66 \pm 0.70$ & 44 & $20.14 \pm 2.38$ & 78 & $19.05 \pm 0.53$ \\
\hline 11 & $19.76 \pm 0.81$ & 45 & $22.49 \pm 0.87$ & 79 & $17.06 \pm 0.69$ \\
\hline 12 & $23.10 \pm 0.50$ & 46 & $21.11 \pm 0.49$ & 80 & $19.98 \pm 1.54$ \\
\hline 13 & $20.31 \pm 0.93$ & 47 & $21.93 \pm 0.97$ & 81 & $20.94 \pm 0.01$ \\
\hline 14 & $17.47 \pm 0.67$ & 48 & $20.40 \pm 0.38$ & 82 & $20.15 \pm 0.64$ \\
\hline 15 & $16.29 \pm 0.95$ & 49 & $20.18 \pm 0.43$ & 83 & $16.15 \pm 0.49$ \\
\hline 16 & $19.55 \pm 1.02$ & 50 & $20.79 \pm 0.90$ & 84 & $20.02 \pm 0.54$ \\
\hline 17 & $16.00 \pm 0.58$ & 51 & $20.99 \pm 1.11$ & 85 & $20.98 \pm 0.59$ \\
\hline 18 & $14.92 \pm 1.16$ & 52 & $15.35 \pm 0.38$ & 86 & $23.40 \pm 0.83$ \\
\hline 19 & $21.25 \pm 0.40$ & 53 & $23.87 \pm 0.77$ & 87 & $23.44 \pm 0.21$ \\
\hline 20 & $21.34 \pm 0.33$ & 54 & $21.28 \pm 1.13$ & 88 & $25.27 \pm 0.64$ \\
\hline 21 & $24.27 \pm 0.64$ & 55 & $14.39 \pm 0.75$ & 89 & $22.75 \pm 0.95$ \\
\hline 22 & $16.62 \pm 0.31$ & 56 & $24.73 \pm 0.73$ & 90 & $17.39 \pm 1.11$ \\
\hline 23 & $20.97 \pm 0.79$ & 57 & $22.46 \pm 1.08$ & 91 & $18.02 \pm 1.62$ \\
\hline 24 & $20.51 \pm 0.84$ & 58 & $19.95 \pm 0.25$ & 92 & $21.97 \pm 1.40$ \\
\hline 25 & $21.32 \pm 0.75$ & 59 & $20.84 \pm 1.09$ & 93 & $16.40 \pm 1.61$ \\
\hline 26 & $23.15 \pm 0.35$ & 60 & $20.93 \pm 1.27$ & 94 & $19.31 \pm 0.35$ \\
\hline 27 & $17.98 \pm 0.58$ & 61 & $22.52 \pm 1.31$ & 95 & $18.24 \pm 1.09$ \\
\hline 28 & $21.39 \pm 0.69$ & 62 & $22.11 \pm 1.23$ & 96 & $16.08 \pm 0.27$ \\
\hline 29 & $19.30 \pm 0.75$ & 63 & $19.32 \pm 1.35$ & 97 & $17.90 \pm 0.65$ \\
\hline 30 & $19.47 \pm 0.47$ & 64 & $20.71 \pm 0.92$ & 98 & $22.07 \pm 1.54$ \\
\hline 31 & $22.74 \pm 0.88$ & 65 & $18.66 \pm 1.65$ & 99 & $23.57 \pm 0.87$ \\
\hline 32 & $20.94 \pm 0.75$ & 66 & $24.58 \pm 1.28$ & 100 & $18.95 \pm 2.12$ \\
\hline 33 & $22.09 \pm 1.09$ & 67 & $21.66 \pm 0.38$ & SC162 & $28.73 \pm 0.27$ \\
\hline 34 & $24.14 \pm 0.56$ & 68 & $17.03 \pm 0.53$ & TWC352 & $27.23 \pm 0.15$ \\
\hline
\end{tabular}


Table 3. Means (M) and their standard error (SE) of 100-grain weight (g) for topcrosses $(T 1=S C 162$ and $T 2=T W C 352)$ under normal condition.

\begin{tabular}{|c|c|c|c|c|c|c|c|c|}
\hline \multirow{2}{*}{ S1-line } & T1 & T2 & \multirow{2}{*}{ S1-line } & T1 & T2 & \multirow{2}{*}{ S1-line } & T1 & T2 \\
\hline & $\mathbf{M} \pm \mathbf{S E}$ & $\mathbf{M} \pm \mathbf{S E}$ & & $\mathbf{M} \pm \mathbf{S E}$ & $\mathbf{M} \pm \mathbf{S E}$ & & $\mathbf{M} \pm \mathbf{S E}$ & $\mathbf{M} \pm$ SE \\
\hline 1 & $23.06 \pm 1.86$ & $23.34 \pm 0.48$ & 35 & $21.70 \pm 1.30$ & $25.01 \pm 0.82$ & 69 & $19.91 \pm 0.52$ & $25.23 \pm 0.49$ \\
\hline 2 & $25.65 \pm 0.68$ & $22.76 \pm 1.24$ & 36 & $22.32 \pm 0.39$ & $22.10 \pm 0.59$ & 70 & $22.16 \pm 0.63$ & $25.39 \pm 0.33$ \\
\hline 3 & $25.42 \pm 0.87$ & $21.73 \pm 0.34$ & 37 & $22.56 \pm 0.62$ & $24.55 \pm 0.24$ & 71 & $20.73 \pm 0.15$ & $23.84 \pm 0.77$ \\
\hline 4 & $25.38 \pm 1.20$ & $23.49 \pm 0.47$ & 38 & $19.40 \pm 0.49$ & $20.87 \pm 0.81$ & 72 & $24.83 \pm 0.84$ & $24.40 \pm 0.51$ \\
\hline 5 & $23.68 \pm 1.60$ & $23.72 \pm 0.29$ & 39 & $21.55 \pm 0.46$ & $23.45 \pm 0.64$ & 73 & $21.13 \pm 0.86$ & $24.80 \pm 0.95$ \\
\hline 6 & $22.49 \pm 0.53$ & $23.35 \pm 0.39$ & 40 & $24.92 \pm 0.76$ & $24.87 \pm 0.74$ & 74 & $22.13 \pm 0.45$ & $24.10 \pm 0.28$ \\
\hline 7 & $21.88 \pm 1.31$ & $24.81 \pm 0.66$ & 41 & $22.08 \pm 0.55$ & $24.55 \pm 0.66$ & 75 & $16.22 \pm 0.64$ & $22.64 \pm 0.95$ \\
\hline 8 & $23.47 \pm 0.47$ & $25.34 \pm 0.41$ & 42 & $23.90 \pm 0.80$ & $24.33 \pm 1.02$ & 76 & $23.19 \pm 0.77$ & $24.94 \pm 0.51$ \\
\hline 9 & $23.70 \pm 1.69$ & $21.54 \pm 0.62$ & 43 & $18.98 \pm 0.93$ & $21.30 \pm 1.60$ & 77 & $22.67 \pm 0.39$ & $23.93 \pm 0.27$ \\
\hline 10 & $25.58 \pm 0.80$ & $25.12 \pm 0.46$ & 44 & $19.72 \pm 0.31$ & $23.33 \pm 0.44$ & 78 & $19.62 \pm 0.65$ & $22.62 \pm 0.36$ \\
\hline 11 & $22.39 \pm 0.35$ & $22.83 \pm 0.46$ & 45 & $22.91 \pm 0.66$ & $23.31 \pm 1.28$ & 79 & $21.51 \pm 0.30$ & $21.52 \pm 0.93$ \\
\hline 12 & $25.15 \pm 1.52$ & $22.57 \pm 0.75$ & 46 & $21.00 \pm 0.58$ & $23.75 \pm 0.31$ & 80 & $23.21 \pm 0.64$ & $25.52 \pm 0.36$ \\
\hline 13 & $25.35 \pm 0.65$ & $23.43 \pm 1.11$ & 47 & $23.87 \pm 0.43$ & $25.89 \pm 0.74$ & 81 & $23.47 \pm 0.46$ & $22.87 \pm 0.31$ \\
\hline 14 & $25.04 \pm 0.18$ & $21.14 \pm 1.38$ & 48 & $20.73 \pm 0.28$ & $25.50 \pm 0.87$ & 82 & $25.92 \pm 0.56$ & $23.97 \pm 0.67$ \\
\hline 15 & $24.53 \pm 1.00$ & $22.24 \pm 0.67$ & 49 & $20.56 \pm 0.18$ & $23.73 \pm 1.12$ & 83 & $19.22 \pm 0.32$ & $23.90 \pm 1.57$ \\
\hline 16 & $25.57 \pm 1.20$ & $22.81 \pm 0.53$ & 50 & $21.79 \pm 0.61$ & $24.45 \pm 0.52$ & 84 & $22.13 \pm 0.95$ & $25.43 \pm 0.35$ \\
\hline 17 & $20.32 \pm 1.39$ & $20.00 \pm 0.86$ & 51 & $23.96 \pm 1.22$ & $24.08 \pm 0.23$ & 85 & $19.52 \pm 1.28$ & $23.66 \pm 0.30$ \\
\hline 18 & $20.34 \pm 0.79$ & $25.70 \pm 0.72$ & 52 & $18.79 \pm 0.96$ & $22.65 \pm 0.60$ & 86 & $23.49 \pm 0.61$ & $25.71 \pm 0.31$ \\
\hline 19 & $23.99 \pm 0.92$ & $24.93 \pm 0.59$ & 53 & $25.46 \pm 0.20$ & $24.06 \pm 1.89$ & 87 & $24.84 \pm 0.71$ & $24.61 \pm 0.74$ \\
\hline 20 & $22.51 \pm 0.55$ & $20.72 \pm 0.71$ & 54 & $22.65 \pm 0.43$ & $24.47 \pm 1.05$ & 88 & $24.27 \pm 0.16$ & $23.13 \pm 0.84$ \\
\hline 21 & $22.35 \pm 0.61$ & $24.19 \pm 0.32$ & 55 & $17.04 \pm 0.79$ & $20.40 \pm 0.70$ & 89 & $24.71 \pm 1.04$ & $25.15 \pm 0.49$ \\
\hline 22 & $21.96 \pm 0.54$ & $25.84 \pm 0.93$ & 56 & $21.57 \pm 0.30$ & $26.07 \pm 0.17$ & 90 & $19.22 \pm 0.47$ & $24.00 \pm 0.67$ \\
\hline 23 & $23.47 \pm 0.55$ & $24.83 \pm 1.25$ & 57 & $21.57 \pm 1.01$ & $24.59 \pm 0.69$ & 91 & $24.11 \pm 0.37$ & $23.66 \pm 0.43$ \\
\hline 24 & $23.52 \pm 0.55$ & $23.97 \pm 0.99$ & 58 & $23.52 \pm 0.89$ & $24.87 \pm 1.16$ & 92 & $22.89 \pm 0.69$ & $25.78 \pm 0.74$ \\
\hline 25 & $22.62 \pm 0.86$ & $23.37 \pm 1.35$ & 59 & $21.18 \pm 1.10$ & $25.86 \pm 0.64$ & 93 & $23.65 \pm 0.33$ & $22.00 \pm 0.96$ \\
\hline 26 & $23.22 \pm 1.10$ & $23.43 \pm 0.58$ & 60 & $20.82 \pm 1.10$ & $25.68 \pm 1.05$ & 94 & $24.42 \pm 0.60$ & $23.23 \pm 0.96$ \\
\hline 27 & $22.97 \pm 1.13$ & $21.61 \pm 0.61$ & 61 & $21.62 \pm 1.23$ & $26.61 \pm 0.38$ & 95 & $22.95 \pm 0.50$ & $20.10 \pm 0.25$ \\
\hline 28 & $22.26 \pm 0.94$ & $23.07 \pm 0.42$ & 62 & $24.44 \pm 0.47$ & $25.19 \pm 0.90$ & 96 & $20.95 \pm 0.88$ & $24.45 \pm 0.50$ \\
\hline 29 & $27.39 \pm 0.05$ & $19.56 \pm 0.45$ & 63 & $22.88 \pm 0.98$ & $24.27 \pm 0.60$ & 97 & $20.72 \pm 1.04$ & $24.73 \pm 1.00$ \\
\hline 30 & $25.56 \pm 0.99$ & $24.97 \pm 0.62$ & 64 & $25.74 \pm 0.87$ & $21.74 \pm 0.96$ & 98 & $24.27 \pm 0.62$ & $25.45 \pm 0.58$ \\
\hline 31 & $22.60 \pm 0.44$ & $26.26 \pm 0.27$ & 65 & $20.37 \pm 0.73$ & $24.27 \pm 1.29$ & 99 & $26.23 \pm 0.72$ & $25.02 \pm 0.62$ \\
\hline 32 & $22.49 \pm 1.45$ & $22.56 \pm 0.85$ & 66 & $26.11 \pm 0.65$ & $25.87 \pm 0.24$ & 100 & $23.58 \pm 0.46$ & $23.73 \pm 0.81$ \\
\hline 33 & $23.21 \pm 1.02$ & $24.69 \pm 0.45$ & 67 & $20.89 \pm 0.71$ & $24.23 \pm 0.29$ & & & \\
\hline 34 & $25.80 \pm 0.77$ & $23.35 \pm 1.05$ & 68 & $18.56 \pm 0.71$ & $23.83 \pm 0.56$ & Mean & 22.68 & 23.83 \\
\hline
\end{tabular}


Website: www.aun.edu.eg/faculty_agriculture/journals_issues_form.php E-mail: ajas@aun.edu.eg

Table 4. Means (M) and their standard error (SE) of 100-grain weight (g) for parents (S1-lines and two testers) under water stress condition.

\begin{tabular}{|c|c|c|c|c|c|}
\hline S1-line & $\mathbf{M} \pm \mathbf{S E}$ & S1-line & $\mathbf{M} \pm \mathbf{S E}$ & S1-line & $\mathbf{M} \pm \mathbf{S E}$ \\
\hline 1 & $17.58 \pm 1.43$ & 35 & $15.11 \pm 0.62$ & 69 & $13.31 \pm 0.07$ \\
\hline 2 & $21.18 \pm 0.84$ & 36 & $17.96 \pm 0.44$ & 70 & $15.64 \pm 0.53$ \\
\hline 3 & $16.56 \pm 1.69$ & 37 & $16.25 \pm 0.82$ & 71 & $17.00 \pm 0.58$ \\
\hline 4 & $19.22 \pm 0.80$ & 38 & $15.19 \pm 0.74$ & 72 & $20.53 \pm 1.62$ \\
\hline 5 & $17.41 \pm 0.76$ & 39 & $18.23 \pm 0.34$ & 73 & $18.77 \pm 1.02$ \\
\hline 6 & $13.53 \pm 0.31$ & 40 & $20.70 \pm 0.70$ & 74 & $19.40 \pm 0.39$ \\
\hline 7 & $18.69 \pm 1.36$ & 41 & $16.51 \pm 0.29$ & 75 & $15.66 \pm 2.39$ \\
\hline 8 & $16.65 \pm 0.34$ & 42 & $18.30 \pm 0.35$ & 76 & $15.93 \pm 0.85$ \\
\hline 9 & $17.21 \pm 0.71$ & 43 & $17.17 \pm 0.44$ & 77 & $15.95 \pm 1.07$ \\
\hline 10 & $18.90 \pm 0.62$ & 44 & $17.69 \pm 1.43$ & 78 & $16.18 \pm 0.48$ \\
\hline 11 & $17.17 \pm 0.44$ & 45 & $15.11 \pm 1.06$ & 79 & $14.41 \pm 1.21$ \\
\hline 12 & $17.33 \pm 0.88$ & 46 & $17.70 \pm 0.25$ & 80 & $16.23 \pm 1.86$ \\
\hline 13 & $19.03 \pm 0.90$ & 47 & $17.08 \pm 0.37$ & 81 & $18.65 \pm 0.36$ \\
\hline 14 & $16.43 \pm 0.25$ & 48 & $17.52 \pm 0.31$ & 82 & $18.23 \pm 0.66$ \\
\hline 15 & $14.46 \pm 0.43$ & 49 & $15.92 \pm 0.89$ & 83 & $12.88 \pm 1.05$ \\
\hline 16 & $17.19 \pm 0.47$ & 50 & $16.70 \pm 0.35$ & 84 & $15.11 \pm 0.95$ \\
\hline 17 & $14.25 \pm 0.67$ & 51 & $17.64 \pm 0.92$ & 85 & $17.31 \pm 0.56$ \\
\hline 18 & $13.11 \pm 1.64$ & 52 & $13.79 \pm 0.62$ & 86 & $22.19 \pm 0.62$ \\
\hline 19 & $19.00 \pm 0.58$ & 53 & $19.91 \pm 0.55$ & 87 & $17.61 \pm 0.21$ \\
\hline 20 & $19.26 \pm 0.51$ & 54 & $18.49 \pm 0.84$ & 88 & $21.49 \pm 1.33$ \\
\hline 21 & $19.80 \pm 1.34$ & 55 & $12.27 \pm 0.97$ & 89 & $20.97 \pm 0.80$ \\
\hline 22 & $15.48 \pm 0.62$ & 56 & $21.13 \pm 0.33$ & 90 & $16.10 \pm 0.95$ \\
\hline 23 & $19.42 \pm 0.78$ & 57 & $18.48 \pm 1.15$ & 91 & $15.36 \pm 1.75$ \\
\hline 24 & $19.60 \pm 0.85$ & 58 & $17.93 \pm 1.12$ & 92 & $18.15 \pm 0.11$ \\
\hline 25 & $20.22 \pm 0.42$ & 59 & $18.43 \pm 0.65$ & 93 & $10.80 \pm 0.47$ \\
\hline 26 & $20.42 \pm 0.56$ & 60 & $17.17 \pm 1.01$ & 94 & $17.22 \pm 0.40$ \\
\hline 27 & $16.04 \pm 0.60$ & 61 & $19.98 \pm 0.65$ & 95 & $15.73 \pm 1.19$ \\
\hline 28 & $19.55 \pm 0.53$ & 62 & $20.13 \pm 1.33$ & 96 & $14.49 \pm 0.36$ \\
\hline 29 & $17.96 \pm 0.85$ & 63 & $16.23 \pm 1.82$ & 97 & $15.18 \pm 0.92$ \\
\hline 30 & $17.55 \pm 0.25$ & 64 & $18.32 \pm 1.24$ & 98 & $19.18 \pm 0.66$ \\
\hline 31 & $20.10 \pm 0.67$ & 65 & $16.62 \pm 1.06$ & 99 & $20.98 \pm 1.09$ \\
\hline 32 & $17.26 \pm 1.22$ & 66 & $21.89 \pm 1.17$ & 100 & $16.19 \pm 1.24$ \\
\hline 33 & $17.20 \pm 0.83$ & 67 & $17.91 \pm 0.59$ & SC162 & $27.47 \pm 0.09$ \\
\hline 34 & $22.98 \pm 0.65$ & 68 & $12.28 \pm 0.50$ & TWC352 & $26.50 \pm 0.17$ \\
\hline
\end{tabular}


Table 5. Means (M) and their standard error (SE) of 100-grain weight (g) for topcrosses $(T 1=S C 162$ and $T 2=T W C 352)$ under water stress condition.

\begin{tabular}{|c|c|c|c|c|c|c|c|c|}
\hline \multirow{2}{*}{ S1-line } & T1 & T2 & \multirow{2}{*}{ S1-line } & T1 & T2 & \multirow{2}{*}{ S1-line } & T1 & T2 \\
\hline & $\mathbf{M} \pm \mathbf{S E}$ & $\mathbf{M} \pm \mathbf{S E}$ & & $\mathbf{M} \pm \mathbf{S E}$ & $\mathbf{M} \pm \mathbf{S E}$ & & $\mathbf{M} \pm \mathbf{S E}$ & $\mathbf{M} \pm \mathbf{S E}$ \\
\hline 1 & $20.28 \pm 0.64$ & $21.66 \pm 0.33$ & 35 & $19.89 \pm 0.94$ & $22.00 \pm 0.90$ & 69 & $19.21 \pm 0.51$ & $22.9 \pm 0.33$ \\
\hline 2 & $23.03 \pm 0.58$ & $20.39 \pm 0.75$ & 36 & $20.42 \pm 0.70$ & $20.36 \pm 0.31$ & 70 & $21.63 \pm 0.75$ & $24.43 \pm 0.25$ \\
\hline 3 & $23.95 \pm 1.23$ & $20.03 \pm 0.21$ & 37 & $21.49 \pm 0.60$ & $22.94 \pm 0.13$ & 71 & $18.77 \pm 0.79$ & $21.83 \pm 0.54$ \\
\hline 4 & $23.09 \pm 0.32$ & $21.53 \pm 0.74$ & 38 & $16.78 \pm 0.61$ & $20.39 \pm 0.49$ & 72 & $23.61 \pm 0.56$ & $23.43 \pm 0.48$ \\
\hline 5 & $20.38 \pm 1.45$ & $21.38 \pm 0.59$ & 39 & $19.40 \pm 0.50$ & $22.38 \pm 0.18$ & 73 & $19.44 \pm 1.10$ & $23.81 \pm 0.98$ \\
\hline 6 & $20.45 \pm 0.55$ & $21.20 \pm 0.53$ & 40 & $22.85 \pm 0.07$ & $21.64 \pm 0.95$ & 74 & $19.66 \pm 1.47$ & $21.79 \pm 0.54$ \\
\hline 7 & $20.50 \pm 0.95$ & $23.17 \pm 0.55$ & 41 & $21.06 \pm 0.96$ & $22.22 \pm 0.54$ & 75 & $15.72 \pm 0.59$ & $20.76 \pm 0.36$ \\
\hline 8 & $22.42 \pm 0.45$ & $21.52 \pm 0.25$ & 42 & $21.19 \pm 1.18$ & $23.23 \pm 1.08$ & 76 & $20.95 \pm 1.28$ & $23.05 \pm 1.78$ \\
\hline 9 & $19.50 \pm 1.28$ & $20.49 \pm 0.58$ & 43 & $17.32 \pm 0.88$ & $20.14 \pm 1.50$ & 77 & $21.34 \pm 0.62$ & $23.19 \pm 0.39$ \\
\hline 10 & $25.07 \pm 0.79$ & $23.69 \pm 0.27$ & 44 & $17.57 \pm 0.34$ & $21.65 \pm 0.26$ & 78 & $17.68 \pm 1.25$ & $21.38 \pm 1.04$ \\
\hline 11 & $20.20 \pm 0.45$ & $21.56 \pm 0.57$ & 45 & $22.25 \pm 0.58$ & $21.23 \pm 0.63$ & 79 & $18.67 \pm 0.80$ & $19.48 \pm 0.93$ \\
\hline 12 & $20.55 \pm 0.61$ & $21.49 \pm 0.81$ & 46 & $19.13 \pm 0.34$ & $21.27 \pm 0.80$ & 80 & $20.40 \pm 0.37$ & $22.97 \pm 0.70$ \\
\hline 13 & $23.19 \pm 0.41$ & $20.76 \pm 0.64$ & 47 & $20.86 \pm 0.80$ & $23.83 \pm 0.74$ & 81 & $21.89 \pm 0.32$ & $20.45 \pm 0.53$ \\
\hline 14 & $22.22 \pm 0.19$ & $18.84 \pm 0.65$ & 48 & $19.32 \pm 0.44$ & $23.57 \pm 0.72$ & 82 & $20.77 \pm 1.18$ & $23.47 \pm 0.70$ \\
\hline 15 & $22.67 \pm 1.20$ & $21.49 \pm 0.59$ & 49 & $17.96 \pm 1.32$ & $22.47 \pm 1.29$ & 83 & $16.15 \pm 1.39$ & $22.78 \pm 1.56$ \\
\hline 16 & $21.12 \pm 0.75$ & $20.51 \pm 0.64$ & 50 & $20.63 \pm 0.62$ & $22.49 \pm 0.70$ & 84 & $20.05 \pm 0.55$ & $24.58 \pm 0.21$ \\
\hline 17 & $19.70 \pm 1.04$ & $19.09 \pm 1.12$ & 51 & $22.29 \pm 0.74$ & $22.27 \pm 0.49$ & 85 & $18.82 \pm 1.17$ & $21.92 \pm 1.13$ \\
\hline 18 & $18.86 \pm 1.13$ & $24.38 \pm 0.76$ & 52 & $15.85 \pm 1.16$ & $21.36 \pm 0.70$ & 86 & $21.27 \pm 0.90$ & $24.08 \pm 0.51$ \\
\hline 19 & $22.06 \pm 0.53$ & $21.73 \pm 0.74$ & 53 & $21.58 \pm 1.79$ & $21.93 \pm 1.83$ & 87 & $21.33 \pm 1.28$ & $23.34 \pm 0.28$ \\
\hline 20 & $19.92 \pm 1.19$ & $20.04 \pm 0.78$ & 54 & $21.60 \pm 0.70$ & $23.36 \pm 1.13$ & 88 & $22.55 \pm 0.61$ & $22.50 \pm 0.78$ \\
\hline 21 & $20.65 \pm 0.60$ & $20.65 \pm 1.35$ & 55 & $15.59 \pm 0.68$ & $18.59 \pm 0.12$ & 89 & $22.51 \pm 0.87$ & $24.32 \pm 0.40$ \\
\hline 22 & $21.01 \pm 0.45$ & $24.05 \pm 0.56$ & 56 & $20.42 \pm 0.32$ & $23.32 \pm 0.43$ & 90 & $18.82 \pm 0.64$ & $22.26 \pm 0.59$ \\
\hline 23 & $20.92 \pm 0.86$ & $21.62 \pm 0.29$ & 57 & $18.64 \pm 0.90$ & $23.22 \pm 0.22$ & 91 & $22.09 \pm 0.34$ & $21.54 \pm 0.58$ \\
\hline 24 & $21.90 \pm 0.15$ & $21.87 \pm 1.44$ & 58 & $20.53 \pm 0.56$ & $23.63 \pm 1.20$ & 92 & $21.21 \pm 0.43$ & $25.07 \pm 0.62$ \\
\hline 25 & $19.79 \pm 0.87$ & $21.32 \pm 0.76$ & 59 & $18.21 \pm 0.92$ & $24.55 \pm 0.56$ & 93 & $21.78 \pm 0.80$ & $21.02 \pm 0.77$ \\
\hline 26 & $21.77 \pm 1.07$ & $21.89 \pm 0.97$ & 60 & $19.40 \pm 0.68$ & $24.11 \pm 0.53$ & 94 & $23.47 \pm 0.68$ & $22.01 \pm 1.15$ \\
\hline 27 & $21.85 \pm 1.03$ & $20.80 \pm 0.65$ & 61 & $20.65 \pm 0.94$ & $25.68 \pm 0.23$ & 95 & $21.88 \pm 0.76$ & $18.53 \pm 0.73$ \\
\hline 28 & $21.65 \pm 0.22$ & $22.01 \pm 0.77$ & 62 & $22.42 \pm 0.57$ & $23.15 \pm 0.49$ & 96 & $19.29 \pm 0.33$ & $22.45 \pm 0.45$ \\
\hline 29 & $23.73 \pm 0.75$ & $18.41 \pm 0.58$ & 63 & $22.13 \pm 1.12$ & $23.32 \pm 0.50$ & 97 & $19.44 \pm 0.84$ & $23.07 \pm 1.25$ \\
\hline 30 & $21.63 \pm 1.40$ & $24.23 \pm 0.40$ & 64 & $22.36 \pm 0.21$ & $21.04 \pm 1.02$ & 98 & $21.99 \pm 0.40$ & $24.85 \pm 0.37$ \\
\hline 31 & $21.55 \pm 0.69$ & $25.29 \pm 0.38$ & 65 & $19.24 \pm 0.36$ & $23.18 \pm 1.39$ & 99 & $23.19 \pm 0.17$ & $23.38 \pm 0.86$ \\
\hline 32 & $19.63 \pm 1.58$ & $21.75 \pm 1.11$ & 66 & $23.49 \pm 0.49$ & $24.20 \pm 0.63$ & 100 & $22.45 \pm 0.47$ & $22.97 \pm 1.06$ \\
\hline 33 & $21.22 \pm 0.86$ & $23.53 \pm 0.23$ & 67 & $19.75 \pm 0.71$ & $22.87 \pm 0.14$ & & & \\
\hline 34 & $24.73 \pm 0.41$ & $21.26 \pm 0.97$ & 68 & $16.95 \pm 1.09$ & $21.3 \pm 0.99$ & Mean & 20.68 & 22.20 \\
\hline
\end{tabular}


Assiut J. Agric. Sci., 51 (1) 2020 (1-25)

ISSN: 1110-0486

Website: www.aun.edu.eg/faculty_agriculture/journals_issues_form.php E-mail: ajas@aun.edu.eg

Table 6. Means (M) and their standard error (SE) of grain yield per plot (g) for parents (S1-lines and two testers) under normal condition.

\begin{tabular}{|c|c|c|c|c|c|}
\hline S1-line & $\mathbf{M} \pm \mathbf{S E}$ & S1-line & $\mathbf{M} \pm \mathbf{S E}$ & S1-line & $\mathbf{M} \pm \mathbf{S E}$ \\
\hline 1 & $688.71 \pm 8.15$ & 35 & $764.00 \pm 7.57$ & 69 & $669.67 \pm 5.49$ \\
\hline 2 & $352.30 \pm 2.06$ & 36 & $362.77 \pm 5.30$ & 70 & $645.00 \pm 4.62$ \\
\hline 3 & $234.67 \pm 8.67$ & 37 & $664.00 \pm 7.23$ & 71 & $664.33 \pm 4.06$ \\
\hline 4 & $311.00 \pm 6.66$ & 38 & $660.67 \pm 5.21$ & 72 & $444.15 \pm 4.91$ \\
\hline 5 & $280.67 \pm 5.49$ & 39 & $762.33 \pm 6.94$ & 73 & $679.00 \pm 5.77$ \\
\hline 6 & $710.23 \pm 5.72$ & 40 & $659.33 \pm 7.51$ & 74 & $684.67 \pm 5.78$ \\
\hline 7 & $704.08 \pm 26.39$ & 41 & $687.33 \pm 5.04$ & 75 & $479.33 \pm 4.63$ \\
\hline 8 & $642.48 \pm 12.00$ & 42 & $643.30 \pm 6.11$ & 76 & $681.33 \pm 4.98$ \\
\hline 9 & $634.00 \pm 4.36$ & 43 & $679.67 \pm 6.64$ & 77 & $175.00 \pm 6.35$ \\
\hline 10 & $260.33 \pm 8.69$ & 44 & $659.00 \pm 5.20$ & 78 & $745.33 \pm 6.06$ \\
\hline 11 & $657.33 \pm 15.72$ & 45 & $364.26 \pm 4.15$ & 79 & $454.76 \pm 4.56$ \\
\hline 12 & $371.06 \pm 5.77$ & 46 & $676.33 \pm 4.63$ & 80 & $346.67 \pm 5.78$ \\
\hline 13 & $666.67 \pm 16.44$ & 47 & $253.33 \pm 3.48$ & 81 & $681.00 \pm 5.20$ \\
\hline 14 & $614.00 \pm 7.21$ & 48 & $272.67 \pm 6.96$ & 82 & $716.00 \pm 6.35$ \\
\hline 15 & $657.08 \pm 4.23$ & 49 & $247.67 \pm 14.15$ & 83 & $461.00 \pm 6.08$ \\
\hline 16 & $375.00 \pm 2.89$ & 50 & $676.33 \pm 5.55$ & 84 & $360.33 \pm 6.64$ \\
\hline 17 & $261.39 \pm 14.21$ & 51 & $367.67 \pm 7.22$ & 85 & $627.33 \pm 4.06$ \\
\hline 18 & $235.67 \pm 7.45$ & 52 & $285.25 \pm 4.84$ & 86 & $372.33 \pm 5.49$ \\
\hline 19 & $365.28 \pm 7.82$ & 53 & $712.33 \pm 6.06$ & 87 & $760.67 \pm 5.21$ \\
\hline 20 & $652.17 \pm 12.83$ & 54 & $422.90 \pm 4.12$ & 88 & $677.33 \pm 7.51$ \\
\hline 21 & $322.90 \pm 13.20$ & 55 & $640.67 \pm 7.22$ & 89 & $272.00 \pm 4.36$ \\
\hline 22 & $270.00 \pm 6.35$ & 56 & $683.22 \pm 4.56$ & 90 & $460.42 \pm 6.43$ \\
\hline 23 & $736.00 \pm 9.54$ & 57 & $715.33 \pm 4.06$ & 91 & $616.33 \pm 3.76$ \\
\hline 24 & $625.00 \pm 14.43$ & 58 & $681.38 \pm 3.52$ & 92 & $747.67 \pm 5.24$ \\
\hline 25 & $323.06 \pm 6.74$ & 59 & $384.33 \pm 7.31$ & 93 & $237.33 \pm 4.91$ \\
\hline 26 & $681.67 \pm 6.98$ & 60 & $669.33 \pm 5.78$ & 94 & $625.00 \pm 4.36$ \\
\hline 27 & $281.33 \pm 9.84$ & 61 & $335.33 \pm 4.91$ & 95 & $342.86 \pm 9.18$ \\
\hline 28 & $343.83 \pm 3.61$ & 62 & $448.52 \pm 6.23$ & 96 & $622.00 \pm 3.46$ \\
\hline 29 & $641.67 \pm 11.57$ & 63 & $233.44 \pm 6.06$ & 97 & $266.00 \pm 5.51$ \\
\hline 30 & $725.00 \pm 7.00$ & 64 & $671.00 \pm 6.93$ & 98 & $710.33 \pm 5.78$ \\
\hline 31 & $727.33 \pm 4.63$ & 65 & $764.67 \pm 4.91$ & 99 & $470.00 \pm 4.62$ \\
\hline 32 & $490.07 \pm 5.31$ & 66 & $672.83 \pm 7.51$ & 100 & $781.33 \pm 5.24$ \\
\hline 33 & $673.33 \pm 5.24$ & 67 & $375.67 \pm 4.63$ & SC162 & $1906.67 \pm 23.33$ \\
\hline 34 & $733.67 \pm 3.48$ & 68 & $782.00 \pm 4.93$ & TWC352 & $1576.67 \pm 18.56$ \\
\hline
\end{tabular}


Table 7. Means (M) and their standard error (SE) of grain yield per plot (g) for top-crosses $(\mathrm{T} 1=\mathrm{SC162}$ and $\mathrm{T} 2=\mathrm{TWC352})$ under normal condition.

\begin{tabular}{|c|c|c|c|c|c|c|c|c|}
\hline \multirow{2}{*}{ S1-line } & T1 & T2 & \multirow{2}{*}{$\begin{array}{l}\text { S1- } \\
\text { line }\end{array}$} & T1 & \multirow{2}{*}{$\begin{array}{c}\mathrm{T2} \\
\mathrm{M} \pm \mathrm{SE}\end{array}$} & \multirow{2}{*}{$\begin{array}{l}\text { S1- } \\
\text { line }\end{array}$} & \multirow{2}{*}{$\frac{\mathrm{T1}}{\mathrm{M} \pm \mathrm{SE}}$} & \multirow{2}{*}{$\begin{array}{c}\mathrm{T2} \\
\mathrm{M} \pm \mathrm{SE}\end{array}$} \\
\hline & $\mathbf{M} \pm \mathbf{S E}$ & $\mathbf{M} \pm \mathbf{S E}$ & & $\mathbf{M} \pm \mathbf{S E}$ & & & & \\
\hline 1 & $958.34 \pm 39.29$ & $1022.96 \pm 93.72$ & 35 & $810.00 \pm 74.97$ & $1126.41 \pm 62.73$ & 69 & $687.26 \pm 27.68$ & $961.64 \pm 61.65$ \\
\hline 2 & $1245.75 \pm 84.60$ & $1007.40 \pm 145.38$ & 36 & $683.97 \pm 18.45$ & $967.74 \pm 143.17$ & 70 & $786.16 \pm 66.66$ & $1145.56 \pm 68.59$ \\
\hline 3 & $1172.25 \pm 106.56$ & $809.22 \pm 106.31$ & 37 & $1105.68 \pm 46.78$ & $1244.77 \pm 65.43$ & 71 & $637.90 \pm 24.33$ & $914.19 \pm 16.41$ \\
\hline 4 & $1091.53 \pm 17.96$ & $699.53 \pm 47.37$ & 38 & $862.05 \pm 67.70$ & $1013.96 \pm 58.14$ & 72 & $777.27 \pm 60.76$ & $927.00 \pm 22.50$ \\
\hline 5 & $943.33 \pm 69.81$ & $696.52 \pm 44.23$ & 39 & $807.38 \pm 18.31$ & $1068.61 \pm 96.36$ & 73 & $636.87 \pm 16.74$ & $916.72 \pm 25.28$ \\
\hline 7 & $936.93 \pm 165.45$ & $954.44 \pm 69.96$ & 41 & $1048.72 \pm 82.35$ & $970.64 \pm 117.47$ & 75 & $665.97 \pm 26.31$ & $994.94 \pm 12.83$ \\
\hline 8 & $954.36 \pm 43.92$ & $997.54 \pm 123.96$ & 42 & $817.33 \pm 54.43$ & $763.38 \pm 11.06$ & 76 & $1012.06 \pm 51.75$ & $1031.81 \pm 34.46$ \\
\hline 9 & $934.20 \pm 112.81$ & $672.78 \pm 30.48$ & 43 & $774.33 \pm 75.46$ & $1084.37 \pm 56.67$ & 77 & $815.11 \pm 44.81$ & $871.78 \pm 21.73$ \\
\hline 10 & $1033.78 \pm 66.93$ & $1020.28 \pm 106.04$ & 44 & $816.57 \pm 56.92$ & $859.12 \pm 59.98$ & 78 & $675.76 \pm 40.55$ & $1134.83 \pm 42.95$ \\
\hline 11 & $1152.63 \pm 109.50$ & $940.20 \pm 24.74$ & 45 & $877.39 \pm 7.52$ & $958.54 \pm 135.22$ & 79 & $934.89 \pm 23.47$ & $1143.05 \pm 73.38$ \\
\hline 15 & $1072.17 \pm 82.80$ & $933.64 \pm 52.45$ & 49 & $730.83 \pm 33.40$ & $1006.11 \pm 21.55$ & 83 & $797.96 \pm 54.64$ & $870.56 \pm 18.32$ \\
\hline 16 & $950.65 \pm 82.22$ & $987.76 \pm 82.67$ & 50 & $649.44 \pm 19.65$ & $964.11 \pm 61.40$ & 84 & $941.48 \pm 82.63$ & $962.11 \pm 57.19$ \\
\hline 17 & $867.22 \pm 108.93$ & $842.57 \pm 37.81$ & 51 & $997.44 \pm 45.08$ & $1000.44 \pm 56.01$ & 85 & $607.57 \pm 64.47$ & $893.33 \pm 54.88$ \\
\hline 18 & $887.36 \pm 71.86$ & $875.22 \pm 54.07$ & 52 & $689.49 \pm 75.56$ & $1102.02 \pm 68.55$ & 86 & $1287.10 \pm 22.52$ & $1166.78 \pm 36.96$ \\
\hline 19 & $1008.36 \pm 91.06$ & $1203.17 \pm 32.56$ & 53 & $915.78 \pm 141.09$ & $906.15 \pm 72.70$ & 87 & $639.50 \pm 92.50$ & $961.50 \pm 74.09$ \\
\hline 20 & $1094.40 \pm 113.03$ & $1016.90 \pm 81.61$ & 54 & $798.08 \pm 81.02$ & $970.08 \pm 22.33$ & 88 & $953.66 \pm 75.97$ & $964.95 \pm 32.66$ \\
\hline 21 & $940.22 \pm 61.08$ & $896.89 \pm 28.88$ & 55 & $830.00 \pm 64.32$ & $946.18 \pm 56.55$ & 89 & $811.93 \pm 84.60$ & $955.54 \pm 82.86$ \\
\hline 22 & $932.88 \pm 96.54$ & $971.68 \pm 61.46$ & 56 & $666.38 \pm 87.88$ & $999.59 \pm 57.96$ & 90 & \begin{tabular}{|l|}
$866.92 \pm 43.21$ \\
\end{tabular} & $1117.21 \pm 71.24$ \\
\hline 23 & $1081.33 \pm 1.33$ & $783.70 \pm 75.52$ & 57 & $919.89 \pm 27.43$ & $997.30 \pm 80.54$ & 91 & \begin{tabular}{|l|}
$915.36 \pm 33.90$ \\
\end{tabular} & $1107.90 \pm 57.36$ \\
\hline 24 & $968.69 \pm 74.90$ & $967.07 \pm 26.99$ & 58 & $1068.94 \pm 102.37$ & $1088.71 \pm 50.14$ & 92 & $811.22 \pm 12.80$ & $1018.89 \pm 22.40$ \\
\hline 32 & $897.49 \pm 19.21$ & $897.64 \pm 16.78$ & 66 & $922.25 \pm 8.52$ & $1021.24 \pm 22.31$ & 100 & $921.33 \pm 70.28$ & $833.21 \pm 6.35$ \\
\hline 33 & $765.23 \pm 46.68$ & $877.93 \pm 67.10$ & 67 & $821.10 \pm 69.33$ & $883.47 \pm 55.35$ & & & \\
\hline 34 & $1060.13 \pm 8.40$ & $1119.17 \pm 81.52$ & 68 & $934.31 \pm 73.98$ & $1101.33 \pm 69.36$ & Mean & 899.20 & 980.72 \\
\hline
\end{tabular}


Website: www.aun.edu.eg/faculty_agriculture/journals_issues_form.php E-mail: ajas@aun.edu.eg

Table 8. Means (M) and their standard error (SE) of grain yield per plot (g) for parents (S1-lines and two testers) under water stress condition.

\begin{tabular}{|c|c|c|c|c|c|}
\hline S1-line & $\mathbf{M} \pm \mathbf{S E}$ & S1-line & $\mathbf{M} \pm \mathbf{S E}$ & S1-line & $\mathbf{M} \pm \mathbf{S E}$ \\
\hline 1 & $246.16 \pm 6.40$ & 35 & $327.83 \pm 6.35$ & 69 & $283.00 \pm 5.20$ \\
\hline 2 & $215.56 \pm 2.42$ & 36 & $287.33 \pm 4.33$ & 70 & $314.83 \pm 34.15$ \\
\hline 3 & $112.83 \pm 5.29$ & 37 & $288.67 \pm 4.98$ & 71 & $382.00 \pm 4.62$ \\
\hline 4 & $117.33 \pm 3.84$ & 38 & $325.67 \pm 8.11$ & 72 & $339.00 \pm 5.51$ \\
\hline 5 & $169.78 \pm 13.83$ & 39 & $383.00 \pm 8.14$ & 73 & $260.89 \pm 5.60$ \\
\hline 6 & $343.89 \pm 14.13$ & 40 & $278.33 \pm 6.36$ & 74 & $322.67 \pm 3.76$ \\
\hline 7 & $251.31 \pm 11.67$ & 41 & $379.67 \pm 4.63$ & 75 & $244.00 \pm 5.51$ \\
\hline 8 & $290.17 \pm 1.59$ & 42 & $256.05 \pm 6.80$ & 76 & $391.33 \pm 3.76$ \\
\hline 9 & $258.81 \pm 7.65$ & 43 & $355.00 \pm 9.54$ & 77 & $146.00 \pm 6.66$ \\
\hline 10 & $159.17 \pm 6.51$ & 44 & $331.50 \pm 9.25$ & 78 & $344.78 \pm 5.00$ \\
\hline 11 & $191.33 \pm 5.93$ & 45 & $253.07 \pm 5.57$ & 79 & $282.86 \pm 5.15$ \\
\hline 12 & $223.33 \pm 7.51$ & 46 & $245.43 \pm 4.91$ & 80 & $232.00 \pm 4.36$ \\
\hline 13 & $373.75 \pm 9.21$ & 47 & $171.44 \pm 4.82$ & 81 & $388.67 \pm 5.78$ \\
\hline 14 & $238.97 \pm 8.89$ & 48 & $122.67 \pm 3.76$ & 82 & $381.00 \pm 5.20$ \\
\hline 15 & $156.63 \pm 7.61$ & 49 & $153.67 \pm 3.48$ & 83 & $214.00 \pm 5.51$ \\
\hline 16 & $255.65 \pm 9.70$ & 50 & $378.67 \pm 5.49$ & 84 & $220.33 \pm 4.91$ \\
\hline 17 & $148.54 \pm 8.58$ & 51 & $126.67 \pm 4.41$ & 85 & $379.00 \pm 5.77$ \\
\hline 18 & $177.87 \pm 10.32$ & 52 & $124.56 \pm 7.87$ & 86 & $263.67 \pm 4.33$ \\
\hline 19 & $220.33 \pm 15.6$ & 53 & $372.67 \pm 4.26$ & 87 & $378.00 \pm 6.35$ \\
\hline 20 & $220.00 \pm 5.77$ & 54 & $280.11 \pm 3.95$ & 88 & $389.00 \pm 4.62$ \\
\hline 21 & $258.33 \pm 10.14$ & 55 & $275.25 \pm 7.23$ & 89 & $113.67 \pm 3.48$ \\
\hline 22 & $182.22 \pm 4.01$ & 56 & $383.00 \pm 7.23$ & 90 & $220.00 \pm 3.61$ \\
\hline 23 & $312.00 \pm 6.24$ & 57 & $361.00 \pm 4.62$ & 91 & $330.67 \pm 4.06$ \\
\hline 24 & $376.11 \pm 8.73$ & 58 & $349.67 \pm 6.36$ & 92 & $387.00 \pm 4.36$ \\
\hline 25 & $279.00 \pm 6.66$ & 59 & $268.33 \pm 4.63$ & 93 & $118.67 \pm 5.55$ \\
\hline 26 & $245.42 \pm 7.92$ & 60 & $234.33 \pm 5.81$ & 94 & $280.33 \pm 5.49$ \\
\hline 27 & $125.56 \pm 2.42$ & 61 & $263.24 \pm 7.43$ & 95 & $234.11 \pm 5.98$ \\
\hline 28 & $261.25 \pm 9.21$ & 62 & $251.67 \pm 4.98$ & 96 & $346.00 \pm 8.08$ \\
\hline 29 & $352.49 \pm 7.70$ & 63 & $116.00 \pm 5.51$ & 97 & $180.11 \pm 8.23$ \\
\hline 30 & $354.58 \pm 9.02$ & 64 & $257.67 \pm 4.91$ & 98 & $386.00 \pm 4.62$ \\
\hline 31 & $364.33 \pm 7.54$ & 65 & $357.33 \pm 7.42$ & 99 & $228.83 \pm 5.05$ \\
\hline 32 & $305.00 \pm 33.18$ & 66 & $368.33 \pm 5.21$ & 100 & $368.67 \pm 4.91$ \\
\hline 33 & $230.48 \pm 5.79$ & 67 & $239.33 \pm 5.49$ & SC162 & $1408.33 \pm 36.32$ \\
\hline 34 & $378.67 \pm 4.67$ & 68 & $379.67 \pm 5.49$ & TWC352 & $1240.00 \pm 21.79$ \\
\hline
\end{tabular}


Table 9. Means (M) and their standard error (SE) of grain yield per plot (g) for top-crosses $(\mathrm{T} 1=\mathrm{SC} 162$ and $\mathrm{T} 2=\mathrm{TWC352})$ under water stress condition.

\begin{tabular}{|c|c|c|c|c|c|c|c|c|}
\hline \multirow{2}{*}{ S1-line } & T1 & T2 & \multirow{2}{*}{$\begin{array}{l}\text { S1- } \\
\text { line }\end{array}$} & \multirow{2}{*}{$\begin{array}{c}\text { T1 } \\
\mathrm{M} \pm \mathrm{SE}\end{array}$} & \multirow{2}{*}{$\begin{array}{l}\mathbf{T 2} \\
\pm \mathrm{SE}\end{array}$} & \multirow{2}{*}{$\begin{array}{l}\text { S1- } \\
\text { line }\end{array}$} & \multirow[t]{2}{*}{ T1 } & \multirow{2}{*}{$\frac{\text { T2 }}{\mathrm{M} \pm \mathrm{SE}}$} \\
\hline & $\mathrm{M} \pm \mathrm{SE}$ & $\mathrm{M} \pm \mathrm{SE}$ & & & & & & \\
\hline 1 & $748.71 \pm 78.03$ & $826.99 \pm 9.22$ & 35 & $572.84 \pm 123.99$ & $869.58 \pm 58.29$ & 69 & $505.76 \pm 75.68$ & $629.72 \pm 15.72$ \\
\hline 2 & \begin{tabular}{|l|l|}
$878.15 \pm 94.40$ \\
\end{tabular} & $681.43 \pm 34.30$ & 36 & \begin{tabular}{|l|}
$502.79 \pm 49.04$ \\
\end{tabular} & $649.33 \pm 37.97$ & 70 & $490.00 \pm 52.73$ & $997.06 \pm 65.24$ \\
\hline 3 & $828.65 \pm 28.52$ & $1.63 \pm 41.30$ & 37 & $4.77 \pm 92.12$ & $783.68 \pm 86.65$ & 71 & $573.14 \pm 22.71$ & $92.23 \pm 35.96$ \\
\hline 4 & $576.90 \pm 24.34$ & $511.67 \pm 60.99$ & 38 & $586.45 \pm 100.84$ & $838.96 \pm 91.94$ & 72 & $790.18 \pm 36.37$ & $768.21 \pm 24.43$ \\
\hline 5 & $625.73 \pm 42.81$ & $568.72 \pm 22.05$ & 39 & $703.89 \pm 39.70$ & $640.56 \pm 31.72$ & 73 & $585.00 \pm 8.39$ & $817.81 \pm 17.46$ \\
\hline 6 & $831.54 \pm 56.66$ & $706.48 \pm 35.45$ & 40 & $89.62 \pm 55.62$ & $844.82 \pm 29.01$ & 74 & $499.29 \pm 60.89$ & $651.21 \pm 14.79$ \\
\hline 7 & $645.07 \pm 129.16$ & $813.00 \pm 77.18$ & 41 & $785.53 \pm 86.28$ & $658.67 \pm 83.82$ & 75 & $549.03 \pm 11.84$ & $601.18 \pm 37.47$ \\
\hline 8 & $703.67 \pm 76.61$ & $611.68 \pm 51.67$ & 42 & $618.72 \pm 99.92$ & $686.01 \pm 47.40$ & 76 & $842.74 \pm 82.46$ & $726.27 \pm 51.71$ \\
\hline 9 & $533.22 \pm 65.79$ & $597.18 \pm 28.69$ & 43 & $588.91 \pm 77.28$ & $810.41 \pm 111.90$ & 77 & $723.65 \pm 77.84$ & $652.92 \pm 42.48$ \\
\hline 10 & $798.89 \pm 104.08$ & $791.46 \pm 55.76$ & 44 & $626.92 \pm 17.05$ & $740.20 \pm 74.07$ & 78 & $452.17 \pm 35.01$ & $830.27 \pm 68.67$ \\
\hline 11 & $851.05 \pm 106.85$ & $848.86 \pm 26.89$ & 45 & $670.00 \pm 13.32$ & $790.62 \pm 84.29$ & 79 & $646.15 \pm 62.26$ & $648.53 \pm 55.14$ \\
\hline 12 & $660.19 \pm 129.26$ & $0.03 \pm 45.66$ & 46 & $700.74 \pm 106.30$ & $722.24 \pm 13.23$ & 80 & $581.34 \pm 41.30$ & $733.59 \pm 47.56$ \\
\hline 13 & $860.47 \pm 62.82$ & $874.33 \pm 41.03$ & 47 & $620.54 \pm 96.91$ & $806.84 \pm 32.66$ & 81 & $594.94 \pm 26.40$ & $553.73 \pm 44.72$ \\
\hline 14 & $668.46 \pm 145.81$ & $678.46 \pm 125.09$ & 48 & $620.13 \pm 27.92$ & $821.21 \pm 98.03$ & 82 & $534.56 \pm 31.65$ & $701.40 \pm 53.74$ \\
\hline 15 & $691.99 \pm 63.09$ & $811.81 \pm 26.68$ & 49 & $432.50 \pm 93.19$ & $707.73 \pm 109.51$ & 83 & $496.99 \pm 35.16$ & $739.00 \pm 33.38$ \\
\hline 16 & $548.75 \pm 51.37$ & $685.78 \pm 63.32$ & 50 & $417.06 \pm 46.58$ & $678.84 \pm 31.78$ & 84 & $696.67 \pm 60.64$ & $664.33 \pm 76.3$ \\
\hline 17 & 649.7 & 27 & 51 & 85 & 49 & 85 & $529.69 \pm 58.12$ & $74.71 \pm 66.95$ \\
\hline 18 & $666.94 \pm 1$ & 49 & 52 & $409.14 \pm 52.48$ & $869.83 \pm 81.72$ & 86 & $1140.00 \pm 20.82$ & $826.00 \pm 49.96$ \\
\hline 19 & $675.35 \pm 31.91$ & $766.37 \pm 75.27$ & 53 & $482.50 \pm 101.89$ & $487.94 \pm 6.50$ & 87 & $324.04 \pm 70.16$ & $628.48 \pm 24.85$ \\
\hline 20 & $757.49 \pm 88.38$ & $783.50 \pm 63.18$ & 54 & $621.01 \pm 33.27$ & $647.66 \pm 18.53$ & 88 & $706.28 \pm 41.82$ & $688.40 \pm 46.10$ \\
\hline 21 & $739.47 \pm 56.10$ & $681.10 \pm 77.25$ & 55 & $681.35 \pm 77.76$ & $687.41 \pm 11.76$ & 89 & $571.69 \pm 51.46$ & $843.44 \pm 29.69$ \\
\hline 22 & $876.54 \pm 96.28$ & $7 \pm 57.16$ & 56 & $531.43 \pm 109.70$ & $733.35 \pm 37.16$ & 90 & $602.67 \pm 43.50$ & $921.44 \pm 42.35$ \\
\hline 23 & $1.86 \pm 48.86$ & 7.60 & 57 & \begin{tabular}{|l|}
$704.30 \pm 52.22$ \\
\end{tabular} & $879.93 \pm 17.67$ & 91 & $780.27 \pm 48.88$ & $567.47 \pm 38.13$ \\
\hline 24 & $660.24 \pm 89.90$ & $782.52 \pm 39.82$ & 58 & $660.39 \pm 88.18$ & $915.00 \pm 50.53$ & 92 & $647.55 \pm 68.14$ & $726.07 \pm 22.37$ \\
\hline 25 & $481.04 \pm 76.79$ & $945.41 \pm 103.93$ & 59 & $653.08 \pm 125.56$ & $868.47 \pm 66.67$ & 93 & $550.35 \pm 44.53$ & $714.09 \pm 54.51$ \\
\hline 26 & $706.21 \pm 22.54$ & $723.33 \pm 73.73$ & 60 & $604.02 \pm 45.98$ & $677.47 \pm 44.33$ & 94 & $844.57 \pm 52.89$ & $701.62 \pm 60.94$ \\
\hline 27 & $462.40 \pm 41.51$ & $554.00 \pm 47.16$ & 61 & $533.71 \pm 87.48$ & $773.11 \pm 78.79$ & 95 & $515.48 \pm 50.70$ & $582.54 \pm 91.00$ \\
\hline 28 & $759.51 \pm 7.06$ & $677.49 \pm 57.85$ & 62 & $657.74 \pm 57.81$ & $752.51 \pm 42.60$ & 96 & $589.74 \pm 15.11$ & $882.22 \pm 30.21$ \\
\hline 29 & $907.77 \pm 127.71$ & $639.24 \pm 40.60$ & 63 & $493.11 \pm 48.21$ & $705.72 \pm 46.90$ & 97 & $599.67 \pm 99.83$ & $966.67 \pm 12.44$ \\
\hline 30 & $833.83 \pm 134.71$ & $865.57 \pm 44.44$ & 64 & $505.13 \pm 150.56$ & $763.39 \pm 70.53$ & 98 & $943.48 \pm 27.40$ & $1126.33 \pm 31.99$ \\
\hline 31 & $646.31 \pm 9.09$ & $804.42 \pm 89.22$ & 65 & $625.25 \pm 51.93$ & $834.32 \pm 42.79$ & 99 & $543.84 \pm 99.45$ & $778.33 \pm 290.26$ \\
\hline 32 & $732.58 \pm 115.28$ & $626.07 \pm 50.00$ & 66 & $840.78 \pm 16.86$ & $733.33 \pm 64.69$ & 100 & $759.70 \pm 77.20$ & $643.17 \pm 26.45$ \\
\hline 33 & $661.43 \pm 29.15$ & $611.00 \pm 88.71$ & 67 & $495.49 \pm 96.25$ & $573.33 \pm 15.81$ & & & \\
\hline 34 & \begin{tabular}{|l|}
$952.18 \pm 64.87$ \\
\end{tabular} & \begin{tabular}{|l|}
$823.99 \pm 82.48$ \\
\end{tabular} & 68 & \begin{tabular}{|l}
$618.97 \pm 74.75$ \\
\end{tabular} & $786.73 \pm 55.51$ & Mean & 654.81 & 739.38 \\
\hline
\end{tabular}

\section{Estimates of general combining ability (GCA) effects}

Estimates of GCA effects for 100 -grain weight under normal condition (Table 10) showed that S1lines 55 and 75 showed negative and significant GCA effects with value of -4.53 and -3.82 , respectively. Conversely, S1-lines 10 and 99 displayed positive and significant GCA effects for 100-GW towards with value of 2.10 and 2.37. For the tester, T2 was the best general combiner, in contrast; T1 was poor general combiner for 100-grain weight. However, under water stress condition, estimates of
GCA effects for 100-grain weight (Table 10) revealed that out of 100 S1-lines studied in line $\times$ tester cross, Similarly, S1-lines 55 and 75 showed negative and significant effects of GCA under water stress with values of -4.35 and -3.20 , respectively. However, S1-lines10 and 66 displayed positive and significant GCA effects for 100-GW towards with values of 2.94 and 2.40 , respectively. For the tester, T2 was the best general combiner while $\mathrm{T} 1$ was poor general combiner for 100-grain weight. Similar to the current findings, positive and negative significant GCA effects 
for 1000 grain weight were reported by Wali et al. (2010).

Under normal condition, estimates of GCA effects for grain yield per plot (Table 11) showed that out of the 100 S1-lines studied in line $x$ tester cross, twenty-eight S1-lines exhibited positive and significant GCA effects while twenty-three S1-lines exhibited negative and significant GCA effects. S1-line 86 exhibited the maximum GCA effect with a value of 286.98, whereas 27 and 45 exhibited the lowest GCA effect with a value of -210.99 . For the tester, T2 was the best general combiner while $\mathrm{T} 1$ was poor general combiner for grain yield per plot. Indicating the existence of the best and the poorest general combiners in the group of S1-lines studied, respectively; in addition, S1-lines identified for good general combining ability could be utilized in maize grain improvement programs for improvement of the traits of interest as these S1-lines have high potential to transfer desirable traits to their cross progenies (Abrha et al. 2013). Both positive and negative GCA effects were reported in maize by several investigators (Ahmed and Saleem 2003). Under water stress condition, estimates of GCA effects for grain yield per plot (Table 11) showed that out of the 100 S1-lines studied in line $\times$ tester cross eighteen S1-lines exhibited positive and significant GCA effects while twenty S1-lines exhibited negative and significant GCA effects. S1-line 98 exhibited the maximum GCA effect with a value of 337.81 , whereas S1-line 87 exhibited the lowest GCA effect with a value of 220.84. For the tester, T2 was the best general combiner in contrast; $\mathrm{T} 1$ was poor general combiner for grain yield per plot. Similar to the current findings, positive and negative significant GCA effects for grain yield per plot were reported by Asif et al. (2014). 
Table 10. General combining ability effects (GCA) for 100-grain weight for 100 S1lines and the two testers (SC162 and TWC352) under normal and water stress conditions.

\begin{tabular}{|c|c|c|c|c|c|c|c|c|}
\hline $\begin{array}{c}\text { S1-lines / } \\
\text { testers }\end{array}$ & 100-GWC & 100-GWS & $\begin{array}{c}\text { S1-lines / } \\
\text { testers }\end{array}$ & 100-GWC & 100-GWS & $\begin{array}{c}\text { S1-lines / } \\
\text { testers }\end{array}$ & 100-GWC & 100-GWS \\
\hline 1 & -0.05 & -0.48 & 35 & 0.10 & -0.50 & 68 & $-2.06^{* *}$ & $-2.32^{* *}$ \\
\hline 2 & 0.95 & 0.27 & 36 & -1.04 & -1.05 & 69 & -0.68 & -0.39 \\
\hline 3 & 0.32 & 0.55 & 37 & 0.30 & 0.77 & 70 & 0.52 & $1.59^{* *}$ \\
\hline 4 & $1.19^{*}$ & 0.87 & 38 & $-3.12^{* *}$ & $-2.86^{* *}$ & 71 & -0.97 & -1.15 \\
\hline 5 & 0.45 & -0.56 & 39 & -0.75 & -0.55 & 72 & $1.36^{*}$ & $2.08^{* *}$ \\
\hline 6 & -0.33 & -0.62 & 40 & $1.64^{* *}$ & 0.80 & 73 & -0.29 & 0.18 \\
\hline 7 & 0.09 & 0.39 & 41 & 0.06 & 0.20 & 74 & -0.14 & -0.72 \\
\hline 8 & 1.15 & 0.53 & 42 & 0.86 & 0.76 & 75 & $-3.82^{* *}$ & $-3.20^{* *}$ \\
\hline 9 & -0.63 & $-1.45^{*}$ & 43 & $-3.12^{* *}$ & $-2.71^{* *}$ & 76 & 0.81 & 0.56 \\
\hline 10 & $2.10^{* *}$ & $2.94^{* *}$ & 44 & $-1.73^{* *}$ & $-1.84^{* *}$ & 77 & 0.05 & 0.82 \\
\hline 11 & -0.64 & -0.56 & 45 & -0.14 & 0.30 & 78 & $-2.13^{* *}$ & $-1.91^{* *}$ \\
\hline 12 & 0.61 & -0.43 & 46 & -0.88 & $-1.24^{*}$ & 79 & $-1.74^{* *}$ & $-2.37^{* *}$ \\
\hline 13 & 1.14 & 0.53 & 47 & $1.63^{* *}$ & 0.90 & 80 & 1.11 & 0.24 \\
\hline 14 & -0.16 & -0.91 & 48 & -0.14 & 0.00 & 81 & -0.09 & -0.27 \\
\hline 15 & 0.13 & 0.64 & 49 & -1.11 & $-1.23^{*}$ & 82 & $1.69^{* *}$ & 0.68 \\
\hline 16 & 0.94 & -0.63 & 50 & -0.13 & 0.12 & 83 & $-1.69^{* *}$ & $-1.98^{* *}$ \\
\hline 17 & $-3.09^{* *}$ & $-2.05^{* *}$ & 51 & 0.77 & 0.84 & 84 & 0.53 & 0.87 \\
\hline 18 & -0.23 & 0.18 & 52 & $-2.53^{* *}$ & $-2.84^{* *}$ & 85 & $-1.66^{* *}$ & -1.08 \\
\hline 19 & $1.21^{*}$ & 0.45 & 53 & $1.51^{*}$ & 0.31 & 86 & $1.35^{*}$ & $1.23^{*}$ \\
\hline 20 & $-1.64^{* *}$ & $-1.46^{*}$ & 54 & 0.31 & 1.04 & 87 & $1.47^{*}$ & 0.89 \\
\hline 21 & 0.02 & -0.79 & 55 & $-4.53^{* *}$ & $-4.35^{* *}$ & 88 & 0.45 & 1.08 \\
\hline 22 & 0.65 & 1.09 & 56 & 0.57 & 0.42 & 89 & $1.68^{* *}$ & $1.97^{* *}$ \\
\hline 23 & 0.90 & -0.17 & 57 & -0.17 & -0.52 & 90 & $-1.64^{* *}$ & -0.90 \\
\hline 24 & 0.50 & 0.44 & 58 & 0.94 & 0.63 & 91 & 0.63 & 0.37 \\
\hline 25 & -0.26 & -0.89 & 59 & 0.26 & -0.06 & 92 & 1.08 & $1.70^{* *}$ \\
\hline 26 & 0.07 & 0.38 & 60 & 0.00 & 0.31 & 93 & -0.43 & -0.04 \\
\hline 27 & -0.96 & -0.12 & 61 & 0.86 & $1.72^{* *}$ & 94 & 0.57 & $1.30^{*}$ \\
\hline 28 & -0.59 & 0.39 & 62 & $1.56^{* *}$ & $1.34^{*}$ & 95 & $-1.73^{* *}$ & $-1.24^{*}$ \\
\hline 29 & 0.22 & -0.37 & 63 & 0.32 & $1.28^{*}$ & 96 & -0.55 & -0.57 \\
\hline 30 & $2.01^{* *}$ & $1.49^{*}$ & 64 & 0.49 & 0.26 & 97 & -0.53 & -0.19 \\
\hline 31 & $1.18^{*}$ & $1.98^{* *}$ & 65 & -0.94 & -0.24 & 98 & $1.61^{* *}$ & $1.98^{* *}$ \\
\hline 32 & -0.73 & -0.75 & 66 & $2.74^{* *}$ & $2.40^{* *}$ & 99 & $2.37^{* *}$ & $1.84^{* *}$ \\
\hline 33 & 0.70 & 0.93 & 67 & -0.69 & -0.13 & 100 & 0.40 & $1.27^{*}$ \\
\hline 34 & $1.32^{*}$ & $1.56^{* *}$ & & & & & & \\
\hline $\begin{array}{l}\text { S.E.(gca for } \\
\text { line) }\end{array}$ & 0.60 & 0.59 & $\mathrm{~T} 1$ (SC 162) & $-0.57^{* *}$ & $-0.76^{* *}$ & $\begin{array}{l}\text { S.E. (gca } \\
\text { for tester) }\end{array}$ & 0.09 & 0.08 \\
\hline $\begin{array}{l}\text { S.E. (gi - } \\
\text { gj)line }\end{array}$ & 0.85 & 0.88 & T2(TWC352) & $0.57^{* *}$ & $0.76^{* *}$ & $\begin{array}{l}\text { S.E. (gi - } \\
\text { gj)tester }\end{array}$ & 0.12 & 0.12 \\
\hline
\end{tabular}

$100-\mathrm{GWC}=100$-grain weight under normal condition, 100-GWS $=100$-grain weight under water stress condition. 
Website: www.aun.edu.eg/faculty_agriculture/journals_issues_form.php E-mail: ajas@aun.edu.eg

Table 11. General combining ability effects (GCA) for grain yield per plot for 100 S1-lines and the two testers (SC162 and TWC352) under normal and water stress conditions.

\begin{tabular}{|c|c|c|c|c|c|c|c|c|}
\hline $\begin{array}{c}\text { S1-lines / } \\
\text { testers }\end{array}$ & GYC & GYS & $\begin{array}{c}\text { S1-lines / } \\
\text { testers }\end{array}$ & GYC & GYS & $\begin{array}{c}\text { S1-lines/ } \\
\text { testers }\end{array}$ & GYC & GYS \\
\hline 1 & 50.70 & 90.76 & 35 & 28.25 & 24.11 & 68 & $77.86^{*}$ & 5.75 \\
\hline 2 & 186.62 & $82.69^{*}$ & 36 & -114.10 & $-121.04 *$ & 69 & $-115.51^{*}$ & -129.36 \\
\hline 3 & 50.78 & 43.05 & 37 & 165.81 & 102.13 & 70 & 25.90 & 46.43 \\
\hline 4 & -44.43 & $-152.81^{* * *}$ & 38 & $115.69^{* *}$ & 15.60 & 71 & $-163.91^{* *}$ & -14.41 \\
\hline 5 & $-120.04^{* *}$ & $-99.88^{*}$ & 39 & -21.40 & -24.88 & 72 & $-87.82^{*}$ & $82.10^{*}$ \\
\hline 6 & 4.49 & 71.91 & 40 & 12.32 & 170.12 & 73 & -163.17 & 4.31 \\
\hline 7 & 5.73 & 31.94 & 41 & $\begin{array}{l}-7.44 \\
\end{array}$ & 25.00 & 74 & $-75.41^{*}$ & -121.85 \\
\hline 8 & 35.99 & -39.42 & 42 & 27.92 & -44.74 & 75 & -109.51 & -121.99 \\
\hline 9 & $-136.47^{*}$ & $-131.90^{*}$ & 43 & $119.61^{*}$ & 2.56 & 76 & $81.97^{*}$ & $87.41^{\circ}$ \\
\hline 10 & $87.07^{*}$ & $98.08^{*}$ & 44 & -39.04 & -13.54 & 77 & $-96.51^{*}$ & -8.81 \\
\hline 11 & $106.46^{* *}$ & $152.86^{* *}$ & 45 & $-210.99^{* *}$ & 33.21 & 78 & -34.66 & -55.88 \\
\hline 12 & 72.52 & 43.01 & 46 & -51.46 & 14.39 & 79 & $99.01^{* 1 *}$ & -49.76 \\
\hline 13 & $92.16^{*}$ & 170.30 & 47 & $175.02^{\prime \prime}$ & 16.60 & 80 & 28.79 & -39.64 \\
\hline 14 & $90.37^{*}$ & -23.64 & 48 & 153.17 & 23.57 & 81 & -27.95 & $-122.77^{*}$ \\
\hline 15 & 62.95 & 54.80 & 49 & $-103.68^{* *}$ & $-126.98^{* * *}$ & 82 & $129.32^{* * *}$ & $-79.12^{*}$ \\
\hline 16 & 29.25 & $-79.83^{*}$ & 50 & -42.39 & $-149.15^{* *}$ & 83 & $-105.70^{* *}$ & $-79.11^{*}$ \\
\hline 17 & $-85.06^{*}$ & -10.31 & 51 & -118.38 & 9.22 & 84 & 11.84 & -16.60 \\
\hline 18 & -58.67 & 32.10 & 52 & 149.69 & -57.62 & 85 & $-189.51^{*}$ & $-94.90^{*}$ \\
\hline 19 & $165.81^{*}$ & 23.76 & 53 & 28.25 & $-211.88^{* *}$ & 86 & 286.98 & 285.90 \\
\hline 20 & $115.69^{* t}$ & 73.40 & 54 & $-114.10^{*}$ & -62.76 & 87 & -139.46 & $-220.84^{*}$ \\
\hline 21 & -21.40 & 13.19 & 55 & $235.27^{* *}$ & -12.72 & 88 & 19.35 & 0.24 \\
\hline 22 & 12.32 & $141.61^{* *}$ & 56 & -1.95 & -64.71 & 89 & -56.23 & 10.47 \\
\hline 23 & $\begin{array}{l}-7.44 \\
\end{array}$ & -42.20 & 57 & -1.96 & 95.01 & 90 & 52.11 & 64.96 \\
\hline 24 & 27.92 & 24.28 & 58 & $81.47^{*}$ & 90.60 * & 91 & 71.67 & -23.23 \\
\hline 25 & $119.61^{*}$ & 16.13 & 59 & 69.72 & 63.68 & 92 & -24.90 & -10.29 \\
\hline 26 & -39.04 & 17.67 & 60 & -149.60 & -56.35 & 93 & 22.97 & -64.88 \\
\hline 27 & $-210.99^{* * *}$ & $-188.90^{* *}$ & 61 & -10.61 & -43.69 & 94 & 35.62 & 76.00 \\
\hline 28 & -51.46 & 21.40 & 62 & $-102.11^{* *}$ & 8.03 & 95 & $-85.70^{*}$ & $-148.09^{*}$ \\
\hline 29 & $175.02^{*}$ & 76.41 & 63 & -21.99 & $-97.68^{*}$ & 96 & -57.76 & 38.89 \\
\hline 30 & $153.17^{\text {th }}$ & $152.60^{*}$ & 64 & -1.15 & -62.84 & 97 & $111.04^{*}$ & $86.07^{*}$ \\
\hline 31 & -103.68 & 28.27 & 65 & 167.04 & 32.69 & 98 & 152.40 & $337.81^{*}$ \\
\hline 32 & -42.39 & -17.78 & 66 & 11.35 & 89.96 & 99 & $91.48^{*}$ & -36.01 \\
\hline 33 & $-118.38^{* *}$ & -60.88 & 67 & -71.49 & $-162.69^{* *}$ & 100 & -62.69 & 4.34 \\
\hline 34 & $149.69^{*+1}$ & $190.98^{*}$ & & & & & & \\
\hline $\begin{array}{l}\text { S.E.(gca } \\
\text { for line) }\end{array}$ & 37.79 & 39.36 & T1(SC 162) & $-40.76^{* *}$ & $-42.29^{* *}$ & $\begin{array}{l}\text { S.E. (gca } \\
\text { for tester) }\end{array}$ & 5.34 & 5.57 \\
\hline $\begin{array}{l}\text { S.E. (gi - } \\
\text { gj) line }\end{array}$ & 53.44 & 55.66 & T2(TWC352) & $40.76^{* *}$ & $42.29^{* *}$ & $\begin{array}{l}\text { S.E. (gi - } \\
\text { gj) tester }\end{array}$ & 7.56 & 7.87 \\
\hline
\end{tabular}

$\mathrm{GYC}=$ Grain yield per plot under normal condition, GYS = Grain yield per plot under water stress condition.

\section{Estimation of specific combining ability (SCA)}

Under normal condition, estimates of SCA effects for 100-grain weight (Table 12), both negative and positive significant estimates were detected among the top-crosses. Topcross including S1-line $29 \times \mathrm{SC} 162$ and S1-line $75 \times$ TWC352 were good specific combiners, whereas, topcross including S1-line $29 \times$ TWC352 and S1-line $75 \times \mathrm{SC} 162$ were poor specific combiners. Under water stress condition, estimates of SCA effects for 100-grain weight (Table 13), both negative and positive and significant estimates of SCA effects were detected among the top-crosses. Top-crosses including S1-line 29× SC162 and S1-line $83 \times$ TWC352 were good specific combiners, however, the top-crosses including S1line $29 \times$ TWC352 and S1-line $83 \times \mathrm{SC} 162$ were poor specific combiners. Crosses with positive and significant SCA effects for this trait are desirable as this trait directly contributes to grain yield of maize. In line 
with the present results, significant SCA effects in maize inbred lines evaluated in line $\mathrm{x}$ tester were reported by other researchers Uddin et al. (2006).

Under normal condition, estimates of SCA effects for grain yield per plot (Table 14), both negative and positive significant estimates of SCA effects were detected among the topcrosses. Top-crosses including S1line $29 \times \mathrm{SC} 162$ and S1-line $78 \times$ TWC352 were good specific combiners, whereas, top-crosses involving S1-line $29 \times$ TWC352 and S1line $78 \times \mathrm{SC} 162$ were poor specific combiners. Under water stress condition, estimates of SCA effects for grain yield per plot are shown in Table (15), both negative and positive and significant estimates were detected among the top-crosses. Topcrosses including S1-line $86 \times \mathrm{SC} 162$ and S1-line $70 \times$ TWC352 were good specific combiners, whereas, the topcrosses including S1-line $86 \times \mathrm{TWC} 352$ and S1-line $70 \times \mathrm{SC} 162$ were poor specific combiners. Abrha et al. (2013) stated that top-crosses with highly positive and significant estimates of SCA effect could be utilizes in maize breeding programs. The results of the current study are partly consistent with the findings of (Iqbal et al. 2007; Rahman et al. 2018) for grain yield in maize.

Table 12. Specific combining ability effects of the 200 top-crosses using two testers (SC162 and TWC352) for 100-grain weight under normal condition.

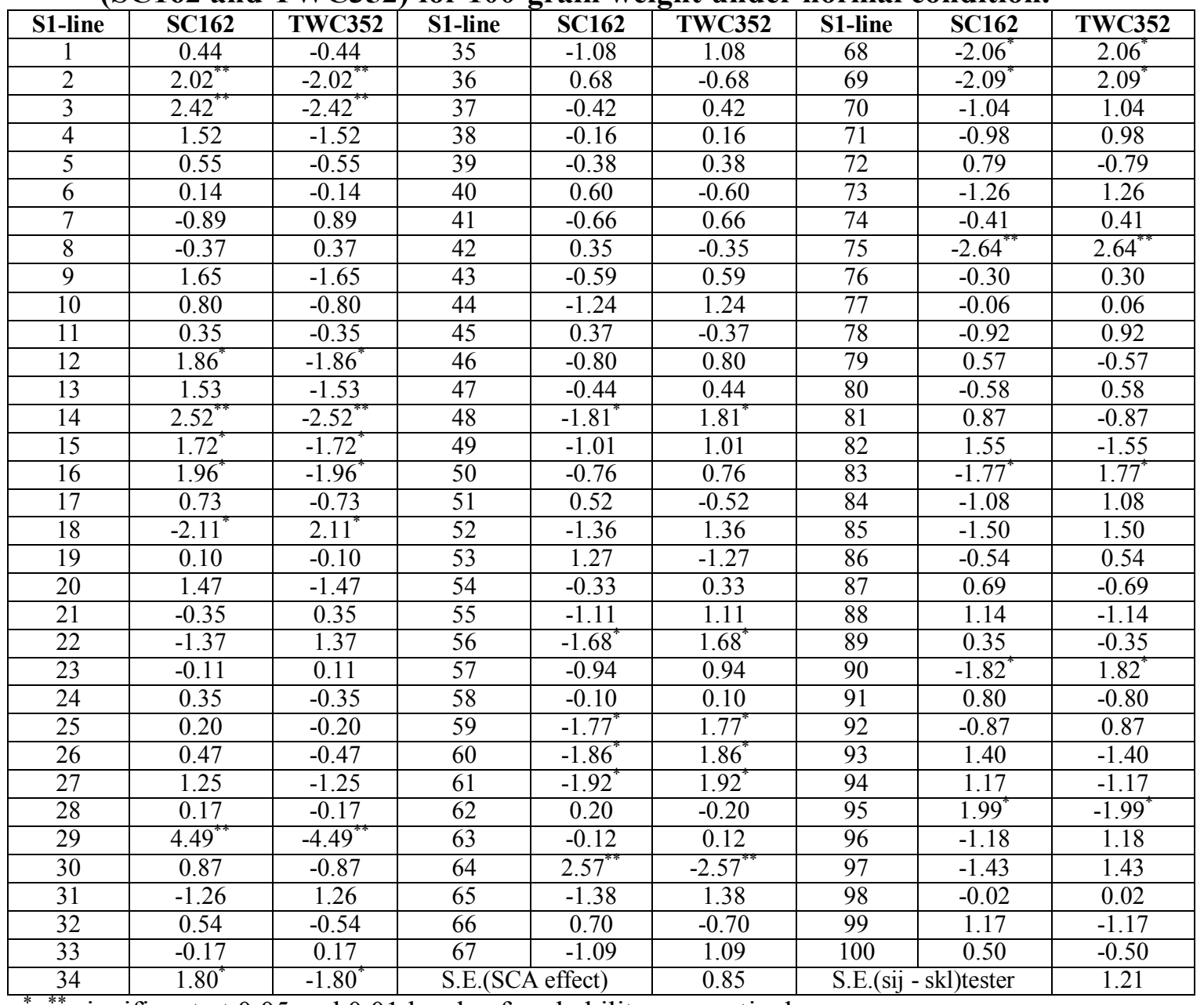

",** significant at 0.05 and 0.01 levels of probability, respectively. 
Website: www.aun.edu.eg/faculty_agriculture/journals_issues_form.php E-mail: ajas@aun.edu.eg

Table 13. Specific combining ability effects of the 200 top-crosses using two testers (SC162 and TWC352) for 100-grain weight under water stress condition.

\begin{tabular}{|c|c|c|c|c|c|c|c|c|}
\hline S1-line & SC162 & TWC352 & S1-line & SC162 & TWC352 & S1-line & SC162 & TWC352 \\
\hline 1 & 0.07 & -0.07 & 35 & -0.30 & 0.30 & 68 & -1.42 & 1.42 \\
\hline 2 & $2.08^{*}$ & $-2.08^{*}$ & 36 & 0.79 & -0.79 & 69 & -1.09 & 1.09 \\
\hline 3 & $2.72^{* *}$ & $-2.72^{* *}$ & 37 & 0.03 & -0.03 & 70 & -0.64 & 0.64 \\
\hline 4 & 1.54 & -1.54 & 38 & -1.05 & 1.05 & 71 & -0.77 & 0.77 \\
\hline 5 & 0.26 & -0.26 & 39 & -0.73 & 0.73 & 72 & 0.85 & -0.85 \\
\hline 6 & 0.38 & -0.38 & 40 & 1.37 & -1.37 & 73 & -1.42 & 1.42 \\
\hline 7 & -0.58 & 0.58 & 41 & 0.18 & -0.18 & 74 & -0.31 & 0.31 \\
\hline 8 & 1.21 & -1.21 & 42 & -0.26 & 0.26 & 75 & $-1.76^{*}$ & $1.76^{*}$ \\
\hline 9 & 0.26 & -0.26 & 43 & -0.65 & 0.65 & 76 & -0.29 & 0.29 \\
\hline 10 & 1.45 & -1.45 & 44 & -1.28 & 1.28 & 77 & -0.16 & 0.16 \\
\hline 11 & 0.08 & -0.08 & 45 & 1.27 & -1.27 & 78 & -1.09 & 1.09 \\
\hline 12 & 0.29 & -0.29 & 46 & -0.31 & 0.31 & 79 & 0.35 & -0.35 \\
\hline 13 & $1.97^{*}$ & $-1.97^{*}$ & 47 & -0.72 & 0.72 & 80 & -0.53 & 0.53 \\
\hline 14 & $2.45^{* *}$ & $-2.45^{* *}$ & 48 & -1.37 & 1.37 & 81 & 1.48 & -1.48 \\
\hline 15 & 1.35 & -1.35 & 49 & -1.50 & 1.50 & 82 & -0.59 & 0.59 \\
\hline 16 & 1.06 & -1.06 & 50 & -0.17 & 0.17 & 83 & $-2.56^{* *}$ & $2.56^{* *}$ \\
\hline 17 & 1.06 & -1.06 & 51 & 0.77 & -0.77 & 84 & -1.50 & 1.50 \\
\hline 18 & $-2.00^{*}$ & $2.00^{*}$ & 52 & $-2.00^{*}$ & $2.00^{*}$ & 85 & -0.79 & 0.79 \\
\hline 19 & 0.92 & -0.92 & 53 & 0.58 & -0.58 & 86 & -0.65 & 0.65 \\
\hline 20 & 0.70 & -0.70 & 54 & -0.12 & 0.12 & 87 & -0.24 & 0.24 \\
\hline 21 & 0.76 & -0.76 & 55 & -0.74 & 0.74 & 88 & 0.79 & -0.79 \\
\hline 22 & -0.76 & 0.76 & 56 & -0.69 & 0.69 & 89 & -0.15 & 0.15 \\
\hline 23 & 0.41 & -0.41 & 57 & -1.53 & 1.53 & 90 & -0.96 & 0.96 \\
\hline 24 & 0.77 & -0.77 & 58 & -0.79 & 0.79 & 91 & 1.04 & -1.04 \\
\hline 25 & 0.00 & 0.00 & 59 & $-2.41^{* *}$ & $2.41^{* *}$ & 92 & -1.17 & 1.17 \\
\hline 26 & 0.70 & -0.70 & 60 & -1.60 & 1.60 & 93 & 1.14 & -1.14 \\
\hline 27 & 1.28 & -1.28 & 61 & $-1.76^{*}$ & $1.76^{*}$ & 94 & 1.49 & -1.49 \\
\hline 28 & 0.58 & -0.58 & 62 & 0.39 & -0.39 & 95 & $2.43^{* *}$ & $-2.43^{* * *}$ \\
\hline 29 & $3.42^{* *}$ & $-3.42^{* *}$ & 63 & 0.16 & -0.16 & 96 & -0.82 & 0.82 \\
\hline 30 & -0.54 & 0.54 & 64 & 1.42 & -1.42 & 97 & -1.05 & 1.05 \\
\hline 31 & -1.11 & 1.11 & 65 & -1.21 & 1.21 & 98 & -0.67 & 0.67 \\
\hline 32 & -0.30 & 0.30 & 66 & 0.41 & -0.41 & 99 & 0.66 & -0.66 \\
\hline 33 & -0.40 & 0.40 & 67 & -0.80 & 0.80 & 100 & 0.50 & -0.50 \\
\hline 34 & $2.49^{* *}$ & $-2.49^{* *}$ & \multicolumn{2}{|c|}{ S.E.(SCA effect) } & 0.84 & \multicolumn{2}{|c|}{ S.E.(sij - skl)tester } & 1.19 \\
\hline
\end{tabular}

, significant at 0.05 and 0.01 levels of probability, respectively. 
Table 14. Specific combining ability effects of the 200 top-crosses using two testers (SC162 and TWC352) for grain yield per plot under normal condition.

\begin{tabular}{|c|c|c|c|c|c|c|c|c|}
\hline S1-line & SC162 & TWC352 & S1-line & SC162 & TWC352 & S1-line & SC162 & TWC352 \\
\hline 1 & 8.45 & -8.45 & 35 & $-117.44^{*}$ & $117.44^{*}$ & 68 & -42.75 & 42.75 \\
\hline 2 & $159.93^{* *}$ & $-159.93^{* *}$ & 36 & -101.12 & 101.12 & 69 & -96.43 & 96.43 \\
\hline 3 & $222.27^{* *}$ & $-222.27^{* *}$ & 37 & -28.78 & 28.78 & 70 & $-138.93^{* *}$ & $138.93^{* *}$ \\
\hline 4 & $236.75^{* *}$ & $-236.75^{* *}$ & 38 & -35.19 & 35.19 & 71 & -97.38 & 97.38 \\
\hline 5 & $164.16^{* *}$ & $-164.16^{* *}$ & 39 & -89.85 & 89.85 & 72 & -34.10 & 34.10 \\
\hline 6 & $115.57^{*}$ & $-115.57^{*}$ & 40 & 26.55 & -26.55 & 73 & -99.16 & 99.16 \\
\hline 7 & 32.00 & -32.00 & 41 & 79.80 & -79.80 & 74 & 17.24 & -17.24 \\
\hline 8 & 19.16 & -19.16 & 42 & 67.73 & -67.73 & 75 & $-123.72^{* *}$ & $123.72^{* *}$ \\
\hline 9 & $171.46^{* *}$ & $-171.46^{* * *}$ & 43 & $-114.26^{*}$ & $114.26^{*}$ & 76 & 30.88 & -30.88 \\
\hline 10 & 47.50 & -47.50 & 44 & 19.48 & -19.48 & 77 & 12.42 & -12.42 \\
\hline 11 & $146.97^{* *}$ & $-146.97^{* *}$ & 45 & 0.18 & -0.18 & 78 & $-188.77^{* *}$ & $188.77^{* *}$ \\
\hline 12 & 3.23 & -3.23 & 46 & 35.52 & -35.52 & 79 & -63.31 & 63.31 \\
\hline 13 & $190.12^{* *}$ & $-190.12^{* * *}$ & 47 & 22.81 & -22.81 & 80 & -79.49 & 79.49 \\
\hline 14 & 35.92 & -35.92 & 48 & 12.67 & -12.67 & 81 & $-120.26^{*}$ & $120.26^{*}$ \\
\hline 15 & $110.02^{*}$ & $-110.02^{*}$ & 49 & -96.88 & 96.88 & 82 & 9.61 & -9.61 \\
\hline 16 & 22.20 & -22.20 & 50 & $-116.57^{*}$ & $116.57^{*}$ & 83 & 4.46 & -4.46 \\
\hline 17 & 53.08 & -53.08 & 51 & 39.25 & -39.25 & 84 & 30.44 & -30.44 \\
\hline 18 & 46.82 & -46.82 & 52 & $-165.50^{* *}$ & $165.50^{* *}$ & 85 & -102.12 & 102.12 \\
\hline 19 & -56.64 & 56.64 & 53 & 45.57 & -45.57 & 86 & 100.91 & -100.91 \\
\hline 20 & 79.50 & -79.50 & 54 & -45.24 & 45.24 & 87 & $-120.24^{*}$ & $120.24^{*}$ \\
\hline 21 & 62.42 & -62.42 & 55 & -17.33 & 17.33 & 88 & 35.11 & -35.11 \\
\hline 22 & 21.35 & -21.35 & 56 & $-125.84^{*}$ & $125.84^{*}$ & 89 & -31.04 & 31.04 \\
\hline 23 & $189.57^{* *}$ & $-189.57^{* *}$ & 57 & 2.05 & -2.05 & 90 & -84.38 & 84.38 \\
\hline 24 & 41.56 & -41.56 & 58 & 30.87 & -30.87 & 91 & -55.51 & 55.51 \\
\hline 25 & -8.67 & 8.67 & 59 & -58.74 & 58.74 & 92 & -63.07 & 63.07 \\
\hline 26 & $115.16^{*}$ & $-115.16^{*}$ & 60 & -104.40 & 104.40 & 93 & -84.31 & 84.31 \\
\hline 27 & $117.05^{*}$ & $-117.05^{*}$ & 61 & $-117.28^{*}$ & $117.28^{*}$ & 94 & 71.00 & -71.00 \\
\hline 28 & 43.22 & -43.22 & 62 & -6.47 & 6.47 & 95 & 101.45 & -101.45 \\
\hline 29 & $303.28^{* *}$ & $-303.28^{* *}$ & 63 & $-115.23^{*}$ & $115.23^{*}$ & 96 & -79.04 & 79.04 \\
\hline 30 & 94.85 & -94.85 & 64 & -61.02 & 61.02 & 97 & -70.24 & 70.24 \\
\hline 31 & -43.29 & 43.29 & 65 & $-185.77^{* *}$ & $185.77^{* *}$ & 98 & -53.59 & 53.59 \\
\hline 32 & 40.68 & -40.68 & 66 & -8.73 & 8.73 & 99 & $-132.24^{*}$ & $132.24^{*}$ \\
\hline 33 & -15.59 & 15.59 & 67 & 9.57 & -9.57 & 100 & 84.82 & -84.82 \\
\hline 34 & 11.24 & -11.24 & \multicolumn{2}{|c|}{ S.E.(SCA effect) } & 53.44 & \multicolumn{2}{|c|}{ S.E.(sij - skl)tester } & 75.57 \\
\hline
\end{tabular}

, significant at 0.05 and 0.01 levels of probability, respectively. 
Website:www.aun.edu.eg/faculty_agriculture/journals_issues_form.php E-mail: ajas@aun.edu.eg

Table 15. Specific combining ability effects of the 200 top-crosses using two testers (SC162 and TWC352) for grain yield per plot under water stress condition.

\begin{tabular}{|c|c|c|c|c|c|c|c|c|}
\hline S1-line & SC162 & TWC352 & S1-line & SC162 & TWC352 & S1-line & SC162 & TWC352 \\
\hline 1 & 3.15 & -3.15 & 35 & -106.08 & 106.08 & 68 & -41.59 & 41.59 \\
\hline 2 & $140.65^{*}$ & $-140.65^{*}$ & 36 & -30.98 & 30.98 & 69 & -19.69 & 19.69 \\
\hline 3 & $130.80^{*}$ & $-130.80^{*}$ & 37 & 57.84 & -57.84 & 70 & $-211.24^{* *}$ & $211.24^{* *}$ \\
\hline 4 & 74.91 & -74.91 & 38 & -83.97 & 83.97 & 71 & -67.25 & 67.25 \\
\hline 5 & 70.79 & -70.79 & 39 & 73.96 & -73.96 & 72 & 53.28 & -53.28 \\
\hline 6 & 104.82 & -104.82 & 40 & 64.69 & -64.69 & 73 & -74.12 & 74.12 \\
\hline 7 & -41.68 & 41.68 & 41 & 105.72 & -105.72 & 74 & -33.67 & 33.67 \\
\hline 8 & 88.29 & -88.29 & 42 & 8.65 & -8.65 & 75 & 16.21 & -16.21 \\
\hline 9 & 10.31 & -10.31 & 43 & -68.46 & 68.46 & 76 & 100.52 & -100.52 \\
\hline 10 & 46.01 & -46.01 & 44 & -14.35 & 14.35 & 77 & 77.66 & -77.66 \\
\hline 11 & 43.38 & -43.38 & 45 & -18.02 & 18.02 & 78 & $-146.76^{* * *}$ & $146.76^{* *}$ \\
\hline 12 & -37.63 & 37.63 & 46 & 31.54 & -31.54 & 79 & 41.10 & -41.10 \\
\hline 13 & 35.36 & -35.36 & 47 & -50.86 & 50.86 & 80 & -33.84 & 33.84 \\
\hline 14 & 37.29 & -37.29 & 48 & -58.25 & 58.25 & 81 & 62.90 & -62.90 \\
\hline 15 & -17.62 & 17.62 & 49 & -95.33 & 95.33 & 82 & -41.13 & 41.13 \\
\hline 16 & -26.22 & 26.22 & 50 & -88.60 & 88.60 & 83 & -78.72 & 78.72 \\
\hline 17 & 5.24 & -5.24 & 51 & 65.04 & -65.04 & 84 & 58.46 & -58.46 \\
\hline 18 & -19.96 & 19.96 & 52 & $-188.06^{* *}$ & 188.06 & 85 & -30.22 & 30.22 \\
\hline 19 & -3.22 & 3.22 & 53 & 39.57 & -39.57 & 86 & $199.29^{* *}$ & $-199.29^{* *}$ \\
\hline 20 & 29.29 & -29.29 & 54 & 28.97 & -28.97 & 87 & -109.93 & 109.93 \\
\hline 21 & 71.48 & -71.48 & 55 & 39.26 & -39.26 & 88 & 51.23 & -51.23 \\
\hline 22 & 80.13 & -80.13 & 56 & -58.67 & 58.67 & 89 & -93.59 & 93.59 \\
\hline 23 & 39.25 & -39.25 & 57 & -45.52 & 45.52 & 90 & -117.10 & 117.10 \\
\hline 24 & -18.85 & 18.85 & 58 & -85.02 & 85.02 & 91 & $148.69^{* *}$ & $-148.69^{* *}$ \\
\hline 25 & $-189.90^{* *}$ & $189.90^{* *}$ & 59 & -65.41 & 65.41 & 92 & 3.03 & -3.03 \\
\hline 26 & 33.73 & -33.73 & 60 & 5.56 & -5.56 & 93 & -39.58 & 39.58 \\
\hline 27 & -3.51 & 3.51 & 61 & -77.41 & 77.41 & 94 & 113.77 & -113.77 \\
\hline 28 & 83.30 & -83.30 & 62 & -5.09 & 5.09 & 95 & 8.76 & -8.76 \\
\hline 29 & $176.56^{* *}$ & $-176.56^{* *}$ & 63 & -64.02 & 64.02 & 96 & -103.95 & 103.95 \\
\hline 30 & 26.42 & -26.42 & 64 & -86.84 & 86.84 & 97 & $-141.21^{*}$ & $141.21^{*}$ \\
\hline 31 & -36.77 & 36.77 & 65 & -62.24 & 62.24 & 98 & -49.14 & 49.14 \\
\hline 32 & 95.55 & -95.55 & 66 & 96.01 & -96.01 & 99 & -74.96 & 74.96 \\
\hline 33 & 67.50 & -67.50 & 67 & 3.37 & -3.37 & 100 & 100.56 & -100.56 \\
\hline 34 & 106.38 & -106.38 & \multicolumn{2}{|c|}{ S.E.(SCA effect) } & 55.66 & \multicolumn{2}{|c|}{ S.E.(sij - skl)tester } & 78.71 \\
\hline
\end{tabular}

, ${ }^{* *}$ significant at 0.05 and 0.01 levels of probability, respectively.

Table 16. Variances of both GCA and SCA along with broad-sense heritability and combining ability ration (CAR) for studied traits under normal and water stress conditions.

\begin{tabular}{|c|c|c|c|c|}
\hline \multirow{2}{*}{} & \multicolumn{2}{|c|}{ Normal condition } & \multicolumn{2}{c|}{ Water stress condition } \\
\cline { 2 - 5 } & $\mathbf{1 0 0 - G W}$ & $\mathbf{G Y}$ & $\mathbf{1 0 0 - G W}$ & $\mathbf{G Y}$ \\
\hline $\mathrm{h}_{\mathrm{b}}$ & 0.7798 & 0.6948 & 0.7521 & 0.7609 \\
\hline$\sigma_{\text {gca }}^{2}$ & 0.0029 & 14.8944 & 0.0057 & 30.2002 \\
\hline$\sigma_{\text {sca }}^{2}$ & 2.5783 & 15777.6400 & 2.1242 & 9800.0770 \\
\hline $\mathrm{CAR}$ & 0.0022 & 0.0018 & 0.0053 & 0.0061 \\
\hline
\end{tabular}

Heritability in broad sense along with variances for both GCA and SCA are shown in Table (16). Briefly, under normal condition heritability in broad sense was higher than under water stress condition for 100 -grain weight, while heritability in broad sense under water stress it was higher than under normal condition for grain yield per plot. Similar results for both traits, (Al-Naggar et al. (2016) found that heritability was increased in stressful environments. In contrast, Worku (2005) reported a 
decrease in heritability under stressed environments.

Under normal and water stress condition, specific combining ability variance was important than general combining ability variance for 100 grain weight and grain yield per plot indicating preponderance of dominance variance in controlling these characters. For both traits, which showed the preponderance of dominance variance, the CAR was lower than unity. Similar results were reported by Emyhum (2013) who found that variance due to SCA was more important than variance due to GCA for 100 -grain weight and grain yield per plot.

\section{Conclusion}

In conclusion, the current study revealed that S1-lines 10, 99, 86, and 55 are good combiners for both 100 grain weight and grain yield per plot based on GCA under normal condition. The top-cross including S1-line $29 \times \mathrm{SC} 162$ is a good specific combiner for both traits under normal condition. Heritability in broad sense showed moderately high estimates for both aforementioned traits. Furthermore, we concluded that the dominance gene action is preponderance in controlling both studied traits. Finally, these S1-lines are promising to produce drought-tolerant inbred lines, which may be used to produce drought tolerant hybrid in the future.

\section{References}

Abrha, S. W., Zeleke, H. Z., \& Gissa, D. W. (2013). Line $\mathrm{x}$ tester analysis of maize inbred lines for grain yield and yield related traits. Asian Journal of Plant Science and Research, 3(5): 12-19.
Ahmad, A., \& Saleem, M. (2003). Combining ability analysis in Zea mays L. Int J Agric Biol, 5: 239-244.

Akula, D., Patil, A. P., Zaidi, P. H., Kuchanur, P., Vi-nayan, M. T., \& Seetharam, K. (2018). Line $\times$ testers analysis of tropical maize inbred lines under heat stress for grain yield and secondary traits. Maydica, 61(1),4-12.

Ali, Q., Ali, A., Ahsan, M., Nasir, I. A., Abbas, H. G., \& Ashraf, M. A. (2014). Line $\times$ Tester analysis for morpho-physiological traits of Zea mays L seedlings. Advancements in Life sciences, 1(4): 242-253.

Ali, R. S. H. (2013). Relationship between combining ability of grain yield and yield components for some newly yellow maize inbred lines via line $\mathrm{x}$ tester analysis. Alex. J. Agric. Res, 58(2): 115124.

Al-Naggar, A. M. M., Atta, M. M. M., Ahmed, M. A., \& Younis, A. S. M. (2016). Influence of deficit condition at silking stage and genotype on maize (Zea mays L.) agronomic and yield characters. Journal of Agriculture and Ecology Research International,5 (2):1-16.

Amin, M. N., Amiruzzaman, M., Ahmed, A., \& Ali, M. R. (2014). Evaluation of inbred lines of maize (Zea mays L.) through line $\times$ tester method. Bangladesh Journal of Agricultural Research, 39(4): 675683.

Asif, A., Liaqat, S., Shah, K. A., \& Shamsur, R. (2014). Heterosis for grain yield and its attributing components in maize variety azam using line $\times$ tester analysis method. Academia Journal of Agricultural Research, 2(11), 225230.

Baker, R.J. (1978). Issues in the diallel analysis. Crop Science, 18: 533536. 
Bartlett, M. S. (1937). "Properties of Sufficiency and Statistical Tests." Proceedings of the Royal Society of London 160:268-282.

Bekele, A., \& Rao, T. N. (2014). Estimates of heritability, genetic advance and correlation study for yield and it's attributes in maize (Zea mays L.). Journal of Plant Sciences, 2(1), 1-4.

De Mendiburu, F. (2014). Agricolae: Statistical Procedures for Agricultural Research. R package version 1.2-2. R Found. Stat. Comput., Vienna.

Emyhum, M. (2013). Estimation of combining ability and heterosis of quality protein maize inbred lines. African Journal of Agricultural Research, 48(8): 6309-6317.

FAOSTAT (2017). Food and Agriculture Organization of the United Nations. Statistics Division, http://faostat3.fao.org/

Hundera, N. B. (2017). Combining ability and heterotic grouping in maize (Zea mays L.) inbred lines for yield and yield related traits. World J Agric Sci, 13: 212-219.

Iqbal, A. M., Nehvi, F. A., Wani, S. A., Qadir, R., \& Dar, Z. A. (2007). Combining ability analysis for yield and yield related traits in maize (Zea mays L.). Int. J. Plant Breed. Genet, 1(1): 101-105.

Kempthorne, O. (1957). An Introduction to Genetic Statistics. John Wiley and sons, inc New York.

Khalili, M., Naghavi, M. R., Aboughadareh, A. P., \& Rad, H. N. (2013). Effects of drought stress on yield and yield components in maize cultivars (Zea mays L.). International Journal of Agronomy and Plant Production; 4(4): 809-812.

Khan, M. B., Hussain, N., \& Iqbal, M. (2001). Effect of water stress on growth and yield components of maize variety YHS 202. Journal of Research Science, 12(1); 15-18.

khattab, S. A., Mustafa, E. A. H., ElEnany, M. A., \& da Siva, J. A. T. (2011). Combining ability for drought tolerance in maize (Zea mays L.) using line $\times$ tester analysis. International Journal of Plant Breeding, 5(2): 122-127.

Ojo, G. O. S., Adedzwa, D. K., \& Bello, L. L. (2007). Combining ability estimates and heterosis for grain yield and yield components in maize (Zea mays L.). Journal of sustainable development in agriculture and environment, 3: 49-57.

Rafiq, C. M., Rafique, M., Hussain, A., \& Altaf, M. (2010). Studies on heritability, correlation and path analysis in maize (Zea mays L.). J. Agric. Res, 48(1): 35-38.

Rahman, M., Rahman, H., Iqbal, M., Khalil, I. H., \& Shah, Z. (2018). Line $\times$ Tester analysis of early generation inbred lines for some agronomic traits in maize. Sarhad Journal of Agriculture, 34(4): 932-940.

Reddy, V. R., Jabeen, F., Sudarshan, M. R., \& Rao, A.S. (2012). Studies on genetic variability, heritability, correlation and path analysis in maize (Zea mays L.) over locations. International Journal of Applied Biology and Pharmaceutical Technology, 4(1):196-199.

Sadalla, H. A., Barznji, M. O., \& Kakarash, S.A. (2017). Full diallel crosses for estimation of genetic parameters in maize. The Iraqi Journal of Agricultural Science, 48 (2), 30-39.

Singh, R. K, D.B. Chaudhary (1985). Biometrical Methods in Quantitative Genetic Analysis. Kalyani Pub. Ludhiana. New Delhi.

Uddin, M. S., Amiruzzaman, M., Bagum, S. A., Hakim, M. A., \& Ali, M. R. (2008). Combining ability and heterosis in maize (Zea mays 
L.). Bangladesh Journal of Plant Breeding and Genetics, 21(1): 2128.

Uddin, M. S., Khatun, F., Ahmed, S., Ali, M. R., \& Bagum, S. A. (2006). Heterosis and combining ability in corn (Zea mays L.). Bangladesh Journal of Botany, 35(2): 109-116.

Wali, M. C., Kachapur, R. M., Chandrashekhar, C. P., Kulkarni, V. R., \& Navadagi, S. D. (2010). Gene action and combining ability studies in single cross hybrids of maize (Zea mays L.). Karnataka Journal of Agricultural Sciences, 23(4): 557-562.

Worku, M. (2005). Genetic and cropphysiological basis of nitrogen efficiency in tropical maize: field studies (Doctoral dissertation, Hannover: Universität).

Yue, H., Chen, S., Bu, J., Wei, J., Peng, H., Li, Y.,... \& Xie, J. (2018). Response of main maize varieties to water stress and comprehensive evaluation in Hebei Province. In IOP Conference Series: Earth and Environmental Science (Vol. 108, No. 4, p.p 042002). IOP Publishing.

Zamaninejad, M., Khorasani, S. K., Moeini, M. J., \& Heidarian, A. R. (2013). Effect of salicylic acid on morphological characteristics, yield and yield components of corn (Zea mays L.) under drought condition. European Journal of Experimental Biology, 3(2):153-161. 
تحليل السلالةxالكثاف للمصول ووزن ال . 1 حبة تحت ظروف الري العادي والجفاف في الأزرة الثامية الصفراء أحت

خليفة علام خليفة سيد'، محمد بدري محمد علي'، خالد عبد الحفيظ محمد إبراهيم'، كمال عبده خير الله'

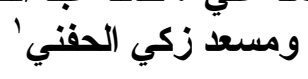

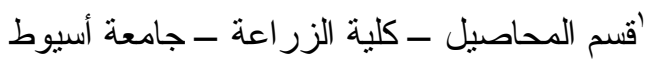

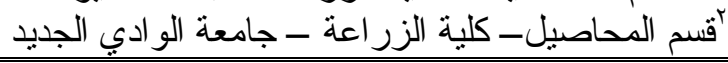

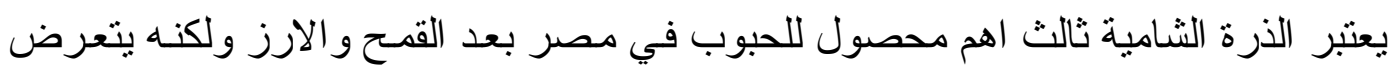

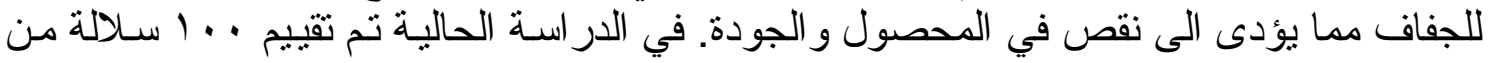

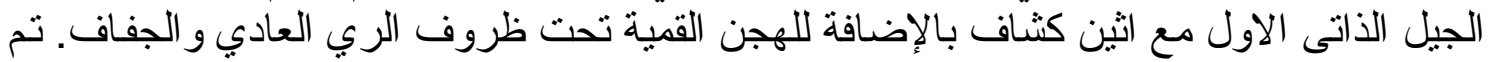

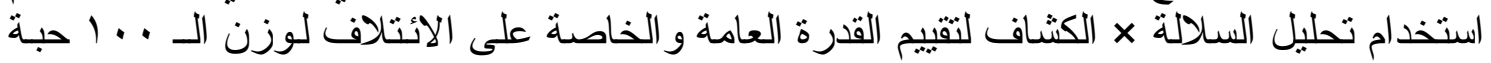

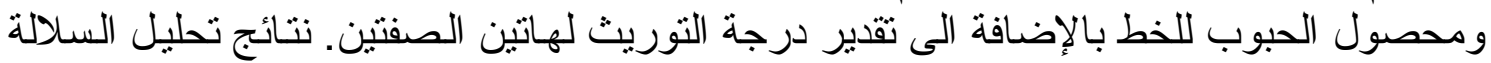

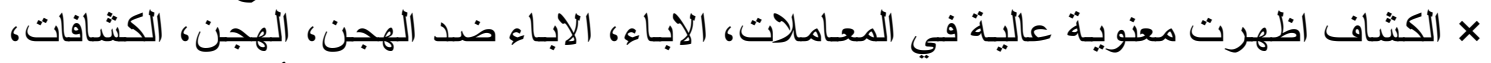

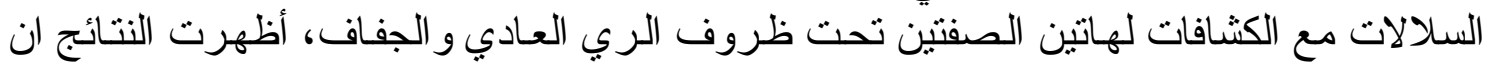

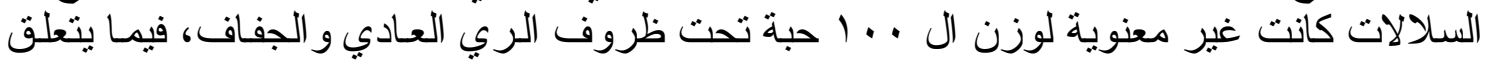

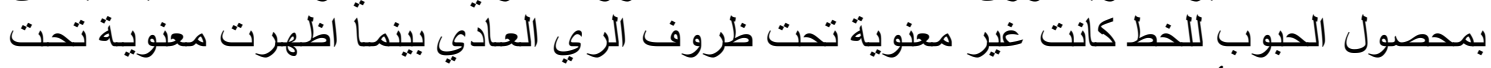

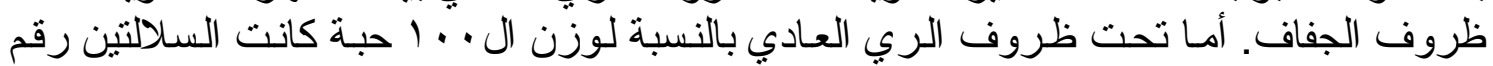

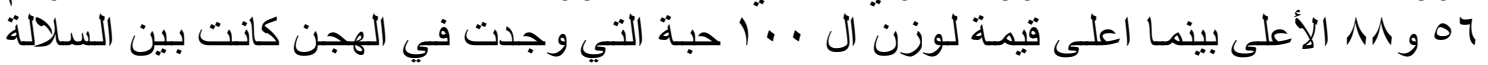

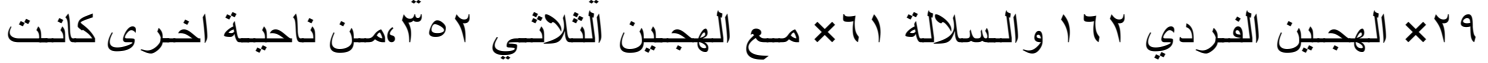

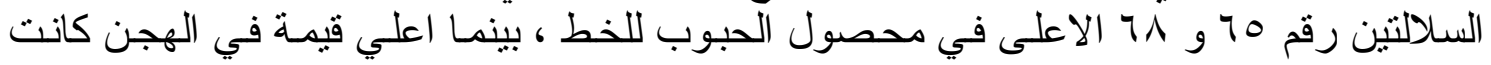

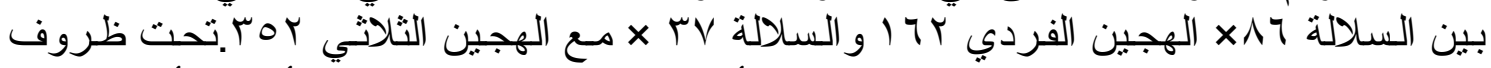

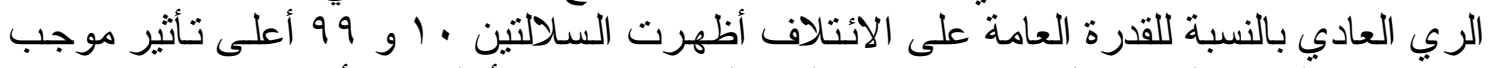

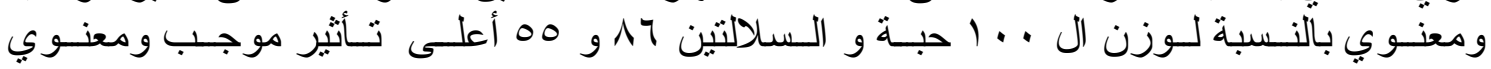

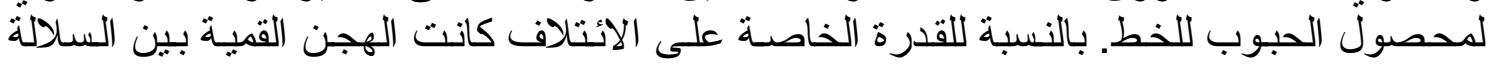

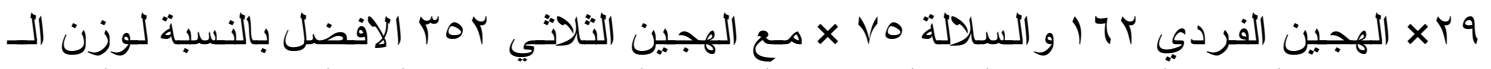

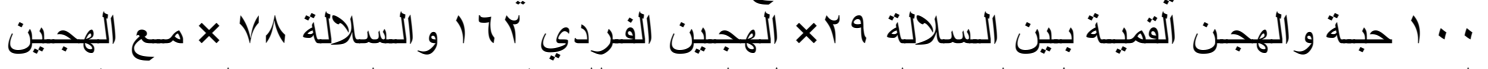

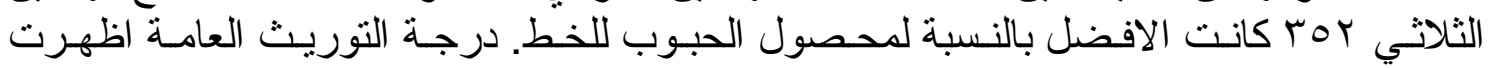

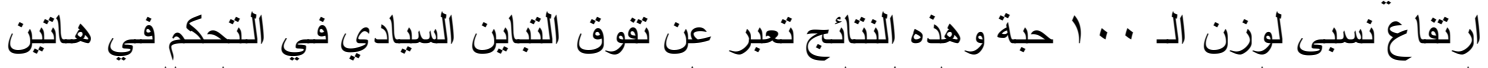

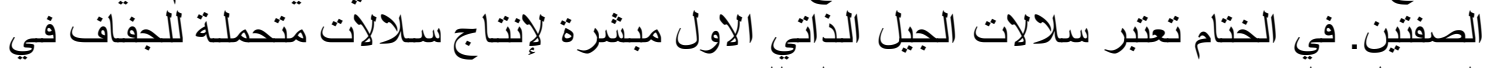
المستقبل و التني تستخدم في انتاج هجن متحملة للجفاف. 